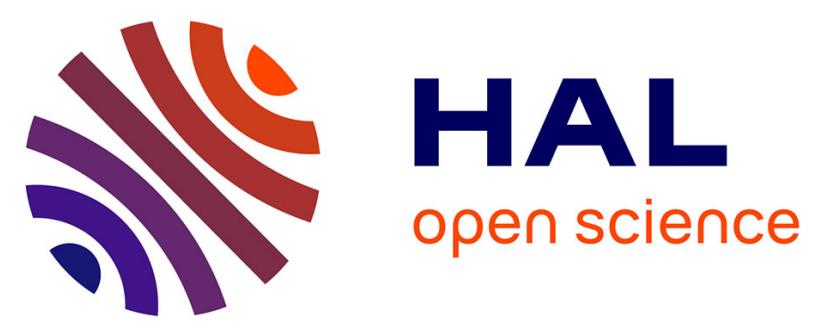

\title{
Dendritic cells require TMEM176A/B ion channels for optimal MHC II antigen presentation to naive CD4 $+\mathrm{T}$ cells
}

Mélanie Lancien, Géraldine Bienvenu, Lucile Guéno, Emmanuel Merieau, Severine Remy, Amandine Even, Aurelie Moreau, Alice Molle, Cynthia Jaeger Fourgeux, Flora Coulon, et al.

\section{To cite this version:}

Mélanie Lancien, Géraldine Bienvenu, Lucile Guéno, Emmanuel Merieau, Severine Remy, et al.. Dendritic cells require TMEM176A/B ion channels for optimal MHC II antigen presentation to naive CD4 $+\mathrm{T}$ cells. 2020. hal-03007864

\section{HAL Id: hal-03007864 https://hal.science/hal-03007864}

Preprint submitted on 16 Nov 2020

HAL is a multi-disciplinary open access archive for the deposit and dissemination of scientific research documents, whether they are published or not. The documents may come from teaching and research institutions in France or abroad, or from public or private research centers.
L'archive ouverte pluridisciplinaire HAL, est destinée au dépôt et à la diffusion de documents scientifiques de niveau recherche, publiés ou non, émanant des établissements d'enseignement et de recherche français ou étrangers, des laboratoires publics ou privés. 


\section{1}

2

3

4

5

6 Melanie Lancien ${ }^{1}$, Geraldine Bienvenu ${ }^{1}$, Lucile Gueno ${ }^{1}$, Sonia Salle ${ }^{1}$, Emmanuel Merieau ${ }^{1}$, Severine

7 Remy $^{1}$, Amandine Even $^{1}$, Aurelie Moreau ${ }^{1}$, Alice Molle ${ }^{1}$, Cynthia Fourgeux ${ }^{1}$, Flora Coulon ${ }^{1}$, Gaelle

8 Beriou $^{1}$, Laurence Bouchet-Delbos ${ }^{1}$, Elise Chiffoleau ${ }^{1}$, Peggy Kirstetter ${ }^{2}$, Susan Chan $^{2}$, Steven

9 Kerfoot $^{3}$, Saeed Abdu Rahiman ${ }^{4}$, Veronica De Simone ${ }^{4}$, Gianluca Matteoli ${ }^{4}$, Gaelle Boncompain ${ }^{5}$,

10 Franck Perez ${ }^{5}$, Regis Josien ${ }^{1}$, Jeremie Poschmann ${ }^{1}$, Maria Cristina Cuturi ${ }^{1,6}$, Cedric Louvet ${ }^{1,6,7, *}$

\section{antigen presentation to naive $\mathrm{CD}^{+} \mathrm{T}$ cells}

\section{Running title : Role of TMEM176A/B in ROR $\gamma \mathbf{t}^{+}$cells and cDCs}

$12{ }^{1}$ Nantes Université, CHU Nantes, Inserm, Centre de Recherche en Transplantation et Immunologie,

13 UMR 1064, ITUN, F-44000 Nantes, France

142 Institut de Génétique et de Biologie Moléculaire et Cellulaire (IGBMC), INSERM U1258, CNRS

15 UMR 7104, Université de Strasbourg, Illkirch, France

$16{ }^{3}$ Department of Microbiology and Immunology, University of Western Ontario, London, Ontario N6A

$175 \mathrm{C} 1$, Canada

$18{ }^{4}$ Department of Chronic Diseases, Metabolism and Ageing, Translational Research Center for

19 Gastrointestinal Disorders (TARGID), University of Leuven, Leuven, Belgium

205 Dynamics of Intracellular Organization Laboratory, Institut Curie, PSL Research University,

21 Sorbonne Université, Centre National de la Recherche Scientifique, UMR 144, Paris, France 
bioRxiv preprint doi: https://doi.org/10.1101/851527; this version posted May 1,2020 . The copyright holder for this preprint (which was not certified by peer review) is the author/funder. All rights reserved. No reuse allowed without permission.

$1{ }^{6}$ These authors contributed equally

\section{$2 \quad{ }^{7}$ Lead Contact}

$3{ }^{*}$ Corresponding author 


\section{$1 \quad$ Summary}

2 Intracellular ion fluxes emerge as critical actors of immunoregulation but still remain poorly explored.

3 Here we investigated the role of the redundant cation channels TMEM176A and TMEM176B

4 (TMEM176A/B) in ROR $\gamma \mathrm{t}^{+}$cells and conventional dendritic cells (cDCs) using germline and

5 conditional double knock-out (DKO) mice. While Tmem176a/b appeared surprisingly dispensable for

6 the protective function of Th17 and group 3 innate lymphoid cells (ILC3s) in the intestinal mucosa, we

7 found that they were required in cDCs for optimal antigen processing and presentation to CD4 ${ }^{+} \mathrm{T}$ cells.

8 Using a real-time imaging method, we show that TMEM176A/B accumulate in dynamic post-Golgi

9 vesicles preferentially linked to the late endolysosomal system and strongly colocalize with HLA-DM.

10 Together, our results suggest that TMEM176A/B ion channels play a direct role in the MHC II

11 compartment (MIIC) of DCs for the fine regulation of antigen presentation and naive $\mathrm{CD}^{+} \mathrm{T}$ cell

12 priming. 


\section{Introduction}

2 Multiple ion channels and transporters are expressed in both innate and adaptive immune cells to control

3 various vital functions, from membrane potential regulation to receptor signaling or migration ${ }^{1}$. The role

4 of ion flux has notably been fully appreciated following the molecular characterization of the store-

5 operated $\mathrm{Ca}^{2+}$ entry (SOCE) through $\mathrm{Ca}^{2+}$ release-activated $\mathrm{Ca}^{2+}(\mathrm{CRAC})$ channels mediated by the

6 ORAI/STIM complex, best characterized in T cells. However, this system appears dispensable for key

7 functions of macrophages and dendritic cells (DCs) while $\mathrm{Ca}^{2+}$ signaling remains critical in these cells $\mathrm{s}^{2,3}$.

8 This observation points to the importance of alternative systems, yet to be discovered, that regulate the

9 intracellular and luminal concentrations of $\mathrm{Ca}^{2+}$ but also other ions including $\mathrm{Na}^{+}, \mathrm{K}^{+}, \mathrm{Cl}^{-}, \mathrm{Mg}^{2+}$ or $\mathrm{Zn}^{2+}$

10 for the control of immune responses. In comparison with the plasma membrane, there is still a paucity

11 of studies investigating the role of intracellular ion channels and transporters, notably in $\mathrm{DCs}^{4}$, that could

12 provide major insights into the understanding of new immunomodulatory mechanisms.

13 We previously showed that the co-regulated genes Tmem176a and Tmem176b encode redundant acidsensitive, non-selective, cation channels ${ }^{5,6}$ whose precise functions remain largely unknown in vivo.

15 Transcriptomic, SNP or epigenetic analysis have associated these homolog genes with different 16 pathologies such as multiple sclerosis ${ }^{7}$, chronic obstructive pulmonary disease (COPD $)^{8}$ or age-related

17 macular degeneration (AMD) ${ }^{9}$. These findings suggest an important role of Tmem176a/b in the

18 development of inflammatory diseases thus emphasizing the need to identify the immune cell types and

19 the functions in which they are predominantly involved.

20 We initially cloned Tmem $176 b$ (originally named Torid) as an over-expressed gene encoding an 21 intracellular four-span transmembrane protein in myeloid cells infiltrating non-rejecting allografts ${ }^{10}$. We

22 later demonstrated its contribution to the suppressive function of ex vivo-generated tolerogenic DCs 23 through antigen cross-presentation by allowing cation $\left(\mathrm{Na}^{+}\right)$counterflux required for progressive 
1 endophagosomal acidification ${ }^{5}$. However, the function of this ion flux in the homeostasis and

2 physiological response of conventional DCs (cDCs) has not been explored and is likely achieved by both

3 TMEM176A and TMEM176B in a redundant fashion ${ }^{6}$.

4 Unexpectedly, besides myeloid cells, we and others reported a strong expression of Tmem $176 a$ and

5 Tmem $176 b$ in the retinoic-acid-receptor-related orphan receptor- $\gamma \mathrm{t}$-positive $\left(\mathrm{ROR} \gamma \mathrm{t}^{+}\right.$) lymphoid cell

6 family, also referred to as type 3 (or type 17) immune cells, producing the prototypical cytokines IL-17

7 and IL-22 and including Th17 CD4 ${ }^{+} \mathrm{T}$ cells, $\gamma \delta \mathrm{T} 17$ cells, group 3 innate lymphoid cells (ILC3s) and

8 NKT17 cells ${ }^{6,11-14}$. Moreover, the Littman group included Tmem176a/b in the restricted group of 11

9 genes whose expression is directly dependent on ROR $\gamma \mathrm{t}$ in Th17 cells ${ }^{15}$. However, in our previous study,

10 we found no or only modest effect of Tmem $176 \mathrm{~b}$ single deficiency in different models of

11 autoinflammation linked to type 3 immunity $^{6}$. We speculated that the absence of Tmem $176 \mathrm{~b}$ could be

12 efficiently compensated by its homolog Tmem176a, located in the same genomic locus, thus masking

13 possible phenotypic alterations.

14 Here we have used germline and conditional ("floxed") double knock-out (DKO) mice to unequivocally

15 determine the importance of Tmem176a and Tmem176b in the biology of ROR $\mathrm{t}^{+}$cells and cDCs in

16 vivo. In that respect, this is the first study exploring the consequence of Tmem $176 a / b$ double deficiency

17 in vivo. Furthermore, we have combined these functional results with the elucidation of the precise

18 trafficking of both proteins using a real-time imaging method. Our findings show that, while

19 Tmem176a/b appear surprisingly dispensable for ROR $\gamma \mathrm{t}^{+}$cell functions, these genes are required in the

20 MHC II pathway in DCs for efficient priming of naive $\mathrm{CD}^{+} \mathrm{T}$ cells. 


\section{$1 \quad$ Results}

2 Generation of germline and conditional double KO (DKO) mice simultaneously targeting Tmem176a and Tmem176b

$4 \quad$ Tmem176a and Tmem176b are homolog genes encoding structurally similar four-span transmembrane

5 proteins $^{6,10,16}$ (Figure 1A). In the immune system, our previous studies ${ }^{6,10,17}$ combined with the analysis

6 of Immuno-Navigator ${ }^{18}$ and Immgen ${ }^{19}$ public databases (Figure S1) indicate that Tmem176a and

$7 \quad$ Tmem $176 b$ are tightly co-regulated and highly expressed both in cDCs and in the ROR $\mathrm{t}^{+}$cell family

8 (depicted in Figure 1B). Taken together, these observations along with reported evidence of genetic

9 compensation in Tmem $176 b^{-/-}$single $\mathrm{KO}$ mice $^{6}$ strongly suggested the need to simultaneously target

10 both genes to decipher their function.

11 As described previously in a methodological study ${ }^{20}$, we generated a germline double KO (DKO) mouse

12 (Figure 1C) in the pure C57BL/6N genetic background. Homozygous DKO mice were born in a

13 Mendelian ratio and appeared normal, without any significant difference in weight from control WT

14 littermates, suggesting that $T m e m 176 a / b$ are not absolutely required, or can be sufficiently compensated,

15 during development.

16 In order to clearly associate a cell type with a potential phenotypic observation resulting from

17 Tmem176a/b deficiency, we also engaged in the generation of a conditional DKO mouse

$18\left(\right.$ Tmem176a/ $\left.b^{\text {flox }}\right)$ (Figure 1D) that we crossed to Rorc $(\gamma \mathrm{t})$-Cre and CD1 1c-Cre (Itgax-Cre) mice to target

$19 \mathrm{ROR}_{\mathrm{t}} \mathrm{t}^{+}$and CD11 $\mathrm{c}^{+}$cells, respectively. Verification of differential Cre-driven cell-specific deletion was

20 performed by comparing $\mathrm{CD}^{+} \mathrm{T}$ cells from the small intestine (si) and splenic cDCs for their expression

21 of Cre, Tmem176a and Tmem $176 b$ mRNA (Figure 1E-F). To increase the fraction of ROR $\gamma \mathrm{t}^{+}$Th17 cells

22 in siCD4 $^{+} \mathrm{T}$ cells, mice were injected with anti-CD3 as previously described ${ }^{21}$. As anticipated, Cre

23 expression was highest in $\operatorname{siCD}^{+} \mathrm{T}$ cells from Rorc-Cre mice and in cDCs from CD11c-Cre mice. 
1 Importantly, both Tmem176a and Tmem176b transcriptional levels were specifically decreased in those

2 subsets as compared to Cre-negative littermate mice. Although deletion efficiency appeared lower in

3 siCD4 $^{+} \mathrm{T}$ cells than in $\mathrm{cDCs}$, this could be explained by the yet relatively low fraction of ROR $\gamma \mathrm{t}^{+}$cells

$4(61.9 \% \pm 7.9)$ within our preparations of purified siCD $4^{+} \mathrm{T}$ cells. Finally, it is interesting to note that

5 cDCs from Rorc-Cre mice exhibited a substantial level of Cre expression, a finding in line with a recent

6 study $^{22}$ describing a subset of cDC expressing Rorc, which however did not appear sufficient to allow

7 Cre-mediated allele recombination.

Tmem176a/b DKO mice exhibit normal ILC3 and Th17 distribution and ROR $\gamma$ cell-dependent

protective functions in the gut mucosa

10 Owing to the high expression of Tmem176a/b in ROR $\gamma \mathrm{t}^{+}$cells, we focused our attention on the intestinal mucosa where these cells preferentially localize to exert their sentinel function in response to the gut microbiota $^{23}$. Flow cytometry analysis (gating strategy depicted in Figure S2A-B) of CD4 ${ }^{+}$T cells and ILCs in the small intestine and colon lamina propria revealed no differences in the proportions of Th17 or ILC3 subsets between WT and DKO mice (Figure 2A). Furthermore, in vitro cytokine production of intestinal $\mathrm{CD}^{+} \mathrm{T}$ cells and ILCs was not affected by Tmem176a/b double deficiency (Figure 2B). Concordant with this, we did not detect any significant change in the level of expression of Rorc2 (the isoform encoding ROR $\gamma \mathrm{t}$ ), Il23r, Il22, Il17 $a$ or $I l 17 f$ as well as target genes of the IL-22/IL17 axis in the small intestine and colon of DKO mice (Figure S2C).

To test whether Tmem $176 a / b$ could play a role in $\mathrm{ROR} \gamma \mathrm{t}^{+}$cells in the context of inflammatory responses in the gut mucosa, we used the injury-induced self-resolving model of dextran sodium sulfate (DSS)induced acute colitis in which Th17/ILC3-derived IL-22 is pivotal to restore barrier integrity upon epithelial damage ${ }^{24,25}$. However, both germline DKO and Tmem176a/ $b^{\mathrm{fl} / \mathrm{fl}}$ Rorc-Cre $^{+/-}$conditional mice 
1 infectious colitis model using Citrobacter rodentium, a mouse attaching and effacing bacterial pathogen

2 considered as an excellent model of clinically important human gastrointestinal pathogens and against

3 which ILC3s and Th17 have been shown to play a protective and redundant role ${ }^{11,26,27}$. As shown in

4 Figure 2D, Tmem 176a/b deficiency did not hamper the mouse resistance to this infection. Moreover,

5 although fecal Citrobacter rodentium bacterial loads detected by qPCR appeared increased in ROR $\gamma \mathrm{t}-$

6 specific conditional DKO, this difference did not reach statistical significance and was not found in

7 germline DKO mice compared to respective control mice (Figure 2E).

8 Thus, these results suggest that Tmem $176 a / b$ are not critical for the development and protective functions

9 of ILC3s and Th17 in the intestinal mucosa.

\section{DCs develop normally in Tmem176a/b-deficient mice}

11 Next, we investigated the effect of Tmem176a/b double deficiency in the biology of cDCs. First, because

12 both genes are highly expressed in the majority of hematopoietic precursors including cDC progenitors

13 (Figure 1B and Figure S1), we assessed their frequency in the bone marrow but found no alteration in

14 DKO mice (Figure 3A and Figure S3). Accordingly, the percentages and absolute numbers of

$15 \mathrm{CD}_{11 \mathrm{c}^{\text {high }}} \mathrm{MHC} \mathrm{II}^{+}$cells in the spleen and, within this population, the proportions of the two main

16 conventional DC subsets, namely $\mathrm{cDC} 1\left(\mathrm{CD} 11 \mathrm{~b}^{-} \mathrm{CD} 8 \alpha^{+}\right)$and $\mathrm{cDC} 2\left(\mathrm{CD} 11 \mathrm{~b}^{+} \mathrm{CD} 8 \alpha^{-}\right)$, were similar

17 between WT and DKO mice (Figure 3B). Furthermore, MHC I, MHC II, CD80 (B7-1) and CD86 (B7-

18 2) surface expression on $\mathrm{cDC} 1$ and $\mathrm{cDC} 2$ were not affected by Tmem $176 a / b$ deficiency and LPS

19 stimulation elicited an equally strong upregulation of MHC II, CD80 and CD86 at the surface of both

20 WT and DKO cDC subsets (Figure 3C). Finally, purified spleen cDCs from WT and DKO mice

21 produced similar basal levels of IL-12 and IL-6 that were both increased with the addition of LPS

22 (Figure 3D). 
1 In the absence of obvious developmental abnormalities of cDCs, we sought to identify dysregulated

2 cellular pathways resulting from the absence of Tmem 176a/b. Chromatin dynamics reflect with great

3 sensitivity gene regulation and play important roles in immune functions such as trained innate

4 immunity $^{28}$ or $\mathrm{T}$ cell effector/memory differentiation ${ }^{29}$. To investigate whether epigenetic alterations are

5 present in DKO cDCs we FACS-sorted $\mathrm{CD} 11 \mathrm{c}^{\text {high }} \mathrm{MHC} \mathrm{II}{ }^{+}$cells and performed chromatin

6 immunoprecipitation by targeting lysine H3K27 acetylation (H3K27ac) followed by sequencing (ChIP-

7 seq) to detect active promoters and enhancers (epigenomics road map). We found that 962 enhancers

8 were significantly differentially acetylated (DA) between WT and DKO cDCs (Figure 3E-F).

9 Interestingly, one of the most significantly DA gene regulatory region covered the entire $C d 74$ gene

10 (Figure 3G) and MHC gene ontology analysis included antigen processing and MHC II protein

11 molecules (Figure 3H). Together, these data suggest that Tmem176a/b deficiency could impact

12 intracellular processes of the MHC II pathway and lead to selective genetic adaptations. Further

13 supporting a role of Tmem176a/b in the MHC II pathway, we found that both homologs were clearly

14 over-expressed in cDC2 when compared to B cells, pDCs and cDC1 (Figure 3I), consistent with the

15 propensity of this subset in priming naive $\mathrm{CD}^{+} \mathrm{T}$ cells through MHC II antigen presentation ${ }^{30}$.

16 Tmem 176a/b are required for optimal presentation of exogenous antigens to CD4 ${ }^{+} \mathrm{T}$ cells by DCs

17 To determine whether Tmem176a/b are required in DCs for antigen presentation in vivo via MHC I or

18 MHC II molecules, we set out to test different models of dominant $\mathrm{CD} 8^{+}$or $\mathrm{CD}^{+} \mathrm{T}$ cell responses.

19 First, using a minor histocompatibility transplantation model in which male skin is grafted onto female

20 recipients, we found that WT and DKO exhibited the same rate of graft rejection (Figure 4A), suggesting

21 that $\mathrm{CD}^{+} \mathrm{T}$ cell priming and effector functions were not impaired in the absence of Tmem176a/b. Next,

22 we examined whether anti-tumor immune responses could be influenced by Tmem176a/b deficiency.

23 Notably, we hypothesized that DKO could exhibit enhanced anti-tumor immunity owing to a recent

Lancien et al. 
1 study proposing that targeting Tmem $176 \mathrm{~b}$ could improve anti-tumor $\mathrm{CD}^{+} \mathrm{T}$-cell response by de-

2 repressing inflammasome activation in myeloid cells ${ }^{31}$. Subcutaneous injection of OVA-expressing EG7

3 thymoma led to detectable tumors within two weeks in all mice and, consistently with the relatively

4 immunogenic nature of this cell line in our experimental conditions, tumor regression was then observed

5 in a large fraction of the mice but without significant difference between WT and DKO mice

6 (Figure 4B). Furthermore, Tmem 176a/b deficiency did not impact tumor growth and mouse survival

7 with two aggressive tumor cell lines, MCA101-sOVA fibrosarcoma and B16-OVA melanoma

8 (Figure 4B). Thus, our results show that the absence of Tmem176a and Tmem176b does not prevent nor

9 enhance $\mathrm{CD}^{+} \mathrm{T}$-cell responses. To evaluate $\mathrm{CD}^{+} \mathrm{T}$-cell responses in vivo, we first used the model of

10 experimental autoimmune-encephalomyelitis (EAE) induced by $\mathrm{MOG}_{35-55}$ peptide or $\mathrm{MOG}_{1-125}$ protein

11 immunization (Figure 4C). Interestingly, whereas EAE developed similarly between WT and DKO

12 mice with MOG peptide immunization (Figure 4D), DKO mice appeared less susceptible if the MOG

13 protein was used (Figure 4E), thus pointing to a specific role of Tmem $176 a / b$ in antigen processing

14 before peptide-MHC II complex display at the surface. Because of the high variability of this model, we

next turned to a model of delayed-type hypersensitivity (DTH) (Figure 4F). Again, we observed that

16 DKO mice differed from WT mice for the DTH response upon challenge in the footpad only if the whole

17 protein was used for the immunization (Figure 4G-H). Taken together, these data suggest that

18 Tmem176a/b deficiency selectively affects the intracellular processing of exogenous antigens for naive

$19 \mathrm{CD}^{+} \mathrm{T}$-cell priming.

20 To further test this hypothesis, we directly assessed antigen-specific T-cell proliferation in WT and DKO 21 mice using naive OVA-specific $\mathrm{CD}^{+}$and $\mathrm{CD}^{+} \mathrm{T}$ cells (Figure 5A). It is important to note that $\mathrm{cDCs}$ 22 are strictly required in this system to induce activation and proliferation of naive $\mathrm{T}$ cells ${ }^{32}$. As shown in

23 Figure 5B-C, $\mathrm{CD}^{+} \mathrm{T}$ cell proliferation was markedly diminished in $\mathrm{DKO}$ mice in comparison to WT 24 mice whereas $\mathrm{CD}^{+}$proliferation was not. Importantly, this alteration was not observed in 
$1 \quad$ Tmem $176 a / b^{\mathrm{fl} / \mathrm{fl}}$ Rorc-Cre ${ }^{+/-}$conditional mice (Figure 5D) but was replicated, although to a lesser extent

2 than in germline DKO mice, in Tmem176a/ $b^{\mathrm{fl} / \mathrm{fl}} C D 11 c-\mathrm{Cre}^{+/-}$mice (Figure 5E).

3 In vitro antigen-specific $\mathrm{CD}^{+} \mathrm{T}$-cell proliferation was also significantly decreased in the absence of

4 Tmem176a/b using GM-CSF-induced bone-marrow derived DCs (BMDCs) only when OVA protein was used (Figure 5F-G), while surface expression of MHC II and co-stimulatory molecules remained

6 unaltered (data not shown).

7 In conclusion, these results show that Tmem $176 a / b$ have an intrinsic function in DCs to allow efficient 8 presentation of exogenous antigens onto MHC II molecules and priming of naive $\mathrm{CD}^{+} \mathrm{T}$ cells.

\section{Tmem 176a/b-deficient cDCs exhibit normal antigen uptake and degradation but dysregulated H2-M}

expression selectively in cDC2

Antigen presentation by MHC II molecules is achieved through a series of complex events (depicted in

Figure 6A) beginning with the uptake and mild degradation of exogenous antigens and including finely regulated processes in the specialized MHC II compartment (MIIC) ${ }^{33}$.

Both OVA endocytosis and degradation were similar in WT and DKO cDCs (Figure 6B), indicating that altered MHC II-mediated antigen presentation by Tmem176a/b DCs cannot be explained by a defect in the initial steps of antigen processing.

17 To determine whether Tmem176a/b deficiency could influence the surface or intracellular levels of key players in the MHC II pathway, we analyzed the expression of MHC II (I-A $\left.{ }^{b}\right), H 2-\mathrm{M}$ and H2-O in splenic B cells, cDC1 and cDC2 by flow cytometry (Figure 6C). As expected, cDC1 exhibited the highest levels of intracellular MHC II, Ii and H2-O whereas H2-M was primarily expressed in cDC2, an equilibrium concordant with the intrinsic efficiency of this subset in MHC II processing ${ }^{30}$. Although we did not detect aberrant expression of these molecules at the surface of DKO cells, we found that, intracellular H2-M was paradoxically and selectively over-expressed in the cDC2 subset of DKO mice 
1 compared to WT mice. Additionally, while Ii (invariant chain) expression was unaltered, the intracellular

2 detection of the MHC II-CLIP peptide complex was not increased at the cell surface but was diminished

3 intracellularly in cDC2 of DKO mice (Figure 6C, lower panels).

4 Taken together, these data suggest that Tmem176a/b-mediated function is directly involved in the MIIC

5 for optimal MHC II antigen loading or trafficking.

6 TMEM176A and TMEM176B traffic in dynamic vesicles between the Golgi apparatus and the

7 endolysosomal compartments

8 To gain insight into the intracellular function of TMEM176A/B ion channels, we aimed to elucidate

9 their subcellular localization that remains elusive as different studies reached different conclusions ${ }^{5,6,34}$.

10 To this end, we used the Retention Using Selective Hooks (RUSH) system ${ }^{35}$, a two-state assay allowing

11 fluorescence-based analysis of intracellular trafficking in living cells at physiological temperature

12 (Figure 7A). We performed dual-color imaging using multiple organelle-specific proteins or probes

13 (Figure 7B) to track the intracellular fate of TMEM176A/B from the endoplasmic reticulum (ER). We

14 used HeLa cells as they allow higher resolution of intracellular compartments compared to immune cells.

15 Addition of biotin triggered a rapid change in the TMEM176B signal from a network of tubular elements

16 characteristic of the ER to a pattern reminiscent of the Golgi apparatus but which rapidly evolved into

17 multiple dynamic vesicles (Figure 7C and Supplemental Video 1). TMEM176A and TMEM176B

18 exhibited a very similar intracellular dynamic, as measured by strong colocalization throughout the time

19 of acquisition (Figure 7D and Supplemental Video 2).

20 Confirming our previous hypothesis that TMEM176B traffics through but does not accumulate in the

21 Golgi apparatus ${ }^{6}$, it clearly separated from Golgin-84, a Golgi-resident protein. After 10-15 min of

22 incubation with biotin, Golgin-84 reached the Golgi apparatus where it stayed after longer incubation

23 time in contrast to TMEM176B (Figure 7E-F and Supplemental Video 3). We then examined a variety 
1 of markers depicted in Figure 7G. We did not, or only marginally, observe accumulation at the plasma

2 membrane, a result that was confirmed by co-imaging with CD44. Interestingly, TMEM176B-bearing

3 vesicles could interact with $\mathrm{RAB}^{+}$early endosomes as well as with $\mathrm{RAB}^{+}$late endosomes. We generated a LAMP1 (CD107a) RUSH construct to best reveal endolysosomes and also observed a strong association with TMEM176B trafficking during the post-Golgi time frame. Of interest, TMEM176B could be found colocalized with TNF or TfR (transferrin receptor), during the late events of endocytosis/recycling of these proteins. However, TMEM176B was not associated with RAB4, a marker of recycling endosomes. Although we cannot rule out alternate recycling pathways, these result suggest that TMEM176A/B follow a relatively selective route among the various vesicular compartments of the cell. In this line, TMEM176B did not traffic through mitochondria labeled by the Mitotracker probe but

was significantly associated with Lysotracker that preferentially marks acidic vesicles. Moreover, we found some colocalization signals using a cation-dependent mannose-6-phosphate receptor (CD-MPR)

RUSH construct but even stronger association with the monomeric clathrin adaptor GGA1, known to decorate the carrier vesicles budding from the TGN and merging toward the endosomes ${ }^{36}$.

Taken together, these data show that TMEM176A/B ion channels preferentially localize in the late endosomal compartment and in vesicular vesicles between the Golgi and the endolysosomal system.

\section{TMEM176B colocalizes with HLA-DM in MHC II-expressing cells}

18 Given the requirement of Tmem 176a/b in MHC II antigen presentation, the selective alteration of H2-M expression in $\mathrm{cDC} 2$ and the preferential trafficking of TMEM176A/B in the late endosomal compartment, we asked whether these ion channels could localize in the MIIC. To recapitulate the MHC II pathway in HeLa cells, we used the HeLa-CIITA cell line that stably expresses the transactivator CIITA $^{37}$. To reveal MHC II (HLA-DR) and HLA-DM localization in these cells, we used plasmid 
1 in Zwarts et al. ${ }^{38}$. Colocalized signals were detected between TMEM176B and HLA-DR, mostly in

2 intracellular vesicles (Figure 7H). However, a more pronounced association was observed with HLA-

3 DM in intracellular compartments presumably highlighting the MIIC.

4 Thus, these results strongly suggest that TMEM176A/B exert their function directly in the MIIC to 5 contribute to efficient MHC II peptide loading and/or trafficking. 


\section{Discussion}

2 Finely tuned ion influx and efflux result from various intricate interplays of multiple channels likely

3 tailored for each type of cell and maturation status. The intriguing high expression of TMEM176A and

4 TMEM176B cation channels in both ROR $\gamma \mathrm{t}^{+}$lymphoid cells and DCs logically raises the question of

5 their specific role in these two very distinct immune cell types. Based on expression and functional data,

6 we reasoned that each gene has the potential to compensate for each other and that simultaneous targeting

7 would be a requisite to avoid such redundancy.

8 We present here the first functional study of Tmem176a/b double KO (DKO) mice, either germline or

9 conditional. Given the broad tissue expression of Tmem176a/b, the "floxed" conditional mouse

10 represents an invaluable tool to achieve Cre-mediated cell-specific deletion and document the role of

11 these ion channels in virtually any tissue or cell of interest. Indeed, there is a growing interest in

12 understanding the role of these homolog genes that are over-expressed in a wide range of cell types other

13 than immune cells including fibroblast $\operatorname{subsets}^{39}$, neurons ${ }^{40}$, adipocytes ${ }^{41}$ or tumor cells ${ }^{42-44}$, likely

14 adapting a universal mechanism of intracellular ion flux regulation to their specific needs. Importantly,

15 these Tmem 176a/b DKO mice were generated directly in a pure genetic background (C57BL/6N) thus

16 avoiding incorrect interpretations resulting from carryover of gene variants of a different background

17 surrounding the targeted locus ${ }^{45,46}$.

18 The striking expression of Tmem $176 a / b$ in all type 3 immune cells and their dependency on the master

19 transcription factor ROR $\gamma \mathrm{t}^{15}$ make these homologs promising candidates to uncover novel aspects of

$20 \mathrm{ROR}_{\mathrm{ft}} \mathrm{t}^{+}$cell biology beyond their cytokine production and could represent a novel therapeutic entry

21 point for treating immune-mediated diseases. However, our results indicate that ROR $\gamma \mathrm{t}$-dependent

22 intestinal repair and host defense functions are not compromised in the absence of Tmem176a/b.

23 Furthermore, the normal development $\mathrm{MOG}_{35-55}$ peptide-induced EAE suggest that these genes are not 
1 required for the pathogenicity of Th17 in this model. Investigating the transcriptomic and epigenetic

2 profiles of purified DKO ILC3s or Th17 cells may expose compensatory mechanisms notably involving

3 the regulation of other ion channels that could sufficiently counterbalance the absence of Tmem $176 a / b$.

4 It is also tempting to speculate that these homologs could be required for IL-17/IL-22-independent

5 functions in $\mathrm{ROR}_{\mathrm{t}} \mathrm{t}^{+}$cells, including the regulation of anti-commensal effector $\mathrm{CD} 4^{+} \mathrm{T}$-cells by ILC3s

6 through MHC II-mediated inhibitory presentation ${ }^{47,48}$. Although Tmem176a/b ${ }^{\mathrm{fl} / \mathrm{fl}}$ Rorc-Cre $^{+/-}$conditional

7 mice did not exhibit increased $\mathrm{CD}^{+} \mathrm{T}$ cell activation and proliferation nor neutrophil accumulation in

8 the colonic lamina propria in comparison to control mice (data not shown), the fact that ILC3s selectively

9 share with DCs the expression of MHC II molecules is in favor of a pivotal role of Tmem 176a/b in this

10 adaptive function.

11 In view of the recent study by Segovia et $a l .{ }^{31}$ using Tmem $176 b$ single KO mice or an ion channel

12 inhibitory molecule, our results do not support the hypothesis that inhibiting TMEM176A/B-mediated

13 ion flux could enhance anti-tumor $\mathrm{CD}^{+} \mathrm{T}$-cell response in vivo. Moreover, we did not observe increased

14 IL-1 $\beta$ production by Tmem 176a/b-deficient BMDCs (data not shown). Although different experimental

15 conditions could explain this discrepancy, one can speculate that TMEM176B must be targeted alone,

16 leaving TMEM176A function intact, to obtain such phenotype, and that the effect of the inhibitory

17 molecule is therefore achieved in a selective manner. Alternatively, because of the 129 genetic

18 background origin of the Tmem $176 b$ single $\mathrm{KO}$ mouse ${ }^{5}$, confounding genetic factors could be invoked,

19 despite $>10$ backcrosses onto the C57BL/6 background and the use of littermates ${ }^{49}$.

20 We initially reported that Tmem $176 a / b$ were highly expressed in cDCs but not in pDCs ${ }^{10,17}$, likely a

21 consequence of E2-2-mediated repression as revealed by Ghosh et al. for Tmem176a ${ }^{50}$. Remarkably,

22 recent mouse and human single-cell RNA-seq analysis highlighted these homologs as markers of

23 selective DC subsets, both in mouse and human ${ }^{51-53}$. Notably, the association of TMEM176B expression 
1 with a subset of $\mathrm{cDC} 2$ in Binnewies et al. ${ }^{53}$ is concordant with our data in the mouse showing that

2 Tmem176a/b are markedly over-expressed in $\mathrm{cDC} 2$ compared to cDC1. cDC2 exhibit an overall

3 dominance in MHC II presentation in vivo resulting from the combination of their intrinsic efficiency ${ }^{30,54}$

4 and their favorable position within lymphoid tissues for antigen uptake ${ }^{55}$. Consistently, we found that

$5 \quad$ Tmem 176a/b deficiency selectively affected the capacity of DCs to prime naive CD4 ${ }^{+} \mathrm{T}$ cells but not

$6 \mathrm{CD}^{+} \mathrm{T}$ cells in vivo. Our results point to a defect in the intracellular processing events for exogenous

7 antigen presentation to MHC II molecules. However, this functional alteration is not complete and it is

8 possible that, in the same manner as in ILC3s, compensatory mechanisms develop in DCs in the absence

9 of Tmem 176a/b. In support of this hypothesis, the fact that H2-M was found selectively over-expressed

10 in $\mathrm{cDC} 2$ of DKO mice suggests an adaptation to alleviate a defect in the MIIC for peptide loading onto

11 MHC II molecules. Alternatively, this expression may also reflect an incorrect intracellular localization

12 of $\mathrm{H} 2-\mathrm{M}$, therefore disrupting the optimal processes leading to MHC II presentation.

13 The analysis of TMEM176A/B intracellular dynamics enabled us to clearly delineate that they

14 preferentially traffic in the late endolysosomal system in close relationship with the Golgi apparatus. In

15 contrast with the limitations in sensitivity and the non-dynamic nature of classical immunostaining, the

16 RUSH system was instrumental in revealing the route taken by TMEM176A/B from the ER and beyond

17 the Golgi apparatus. However, because we focused on the first hour of trafficking after release in most

18 of our analyses, we cannot exclude that TMEM176A/B can eventually reach other compartments over

19 time.

20 The strong colocalization found with HLA-DM in HeLa-CIITA cells supports the hypothesis of a direct

21 role in the MIIC. TMEM176A/B-mediated cation $\left(\mathrm{Na}^{+}\right)$efflux could participate in the regulated

22 acidification of this compartment as a counterion conductance ${ }^{56}$. TMEM176A/B could be located on the

23 limiting membrane of the MIIC or on intraluminal vesicles (ILVs) where a direct interaction with HLA-

24 DM would be possible. In this regard, independently of their ion channel function, it is conceivable that

Lancien et al. 
1 these four-span transmembrane proteins act similarly to tetraspanin molecules to stabilize DM-MHC II

2 interaction ${ }^{57}$. On the same note, given the reported genetic association between TMEM176A and HDL

3 cholesterol levels in human ${ }^{58}, \mathrm{TMEM}$ 176A/B function may be connected to cholesterol-containing

4 microdomains for efficient MHC II trafficking 59 . High resolution imaging, FRET analysis or the

5 characterization of organelle-specific disruption in DKO DCs could be informative to uncover the

6 precise role of TMEM176A/B in the MHC II pathway.

7 In conclusion, while the intrinsic function of TMEM176A/B in $\mathrm{ROR} \gamma \mathrm{t}^{+}$cells remains to be further

8 explored, we found that these cation channels play a substantial role in the MHC II pathway to ensure

9 optimal naive $\mathrm{CD}^{+} \mathrm{T}$ cell priming by DCs. Remarkably, a recent study by the Rudensky group identified

10 these genes by single-cell RNA sequencing as markers of the T-bet ${ }^{-}$cDC2B subset both in mouse and

11 human ${ }^{22}$, a finding that reinforces the hypothesis of a predominant role of TMEM176A/B in cDC2s.

12 Together, these results also suggest that the generation of a Tmem $176 a / b$ reporter mouse could represent

13 an invaluable tool to study cDC2 subsets in vivo. 


\section{Materials and Methods}

\section{Mice}

3 Tmem 176a/b double KO (DKO) mice were generated by a dual targeting approach using the CRISPR-

4 Cas9 system as previously described ${ }^{20}$. DKO mice used in this study were generated in the C57BL/6N

5 genetic background. Three consecutive backcrosses with $\mathrm{C} 57 \mathrm{BL} / 6 \mathrm{~N}$ mice were performed before

6 intercrossing heterozygous mice. To control for cage-dependent microbiota variations, WT and DKO

7 mice were systematically co-housed directly after weaning following sex- and age-matching.

8 Conditional DKO mouse carrying a « floxed» Tmem176a/b allele $\left(\right.$ Tmem $\left.176 a / b^{\mathrm{fl}}\right)$ were generated at

9 Mouse Clinical Institute (Illkirch, France). Briefly, two consecutive rounds of ES cell (C57BL/6N

10 genetic background) modifications using two independent selection cassettes were realized to insert

11 LoxP sites on both sides of Tmem176a and Tmem176b first coding exons. F0 mouse chimera were

12 crossed to a FlpO deleter mouse ${ }^{60}$ (pure C57BL/6N background) to remove the FRT- and F3-flanked

13 Neomycin and Hygromycin selection cassettes abutted to the two LoxP sites. Allele transmission was

14 verified on F1 mice before rederivation and housing in a SPF mouse facility. Tmem $176 a / b^{\mathrm{fl} / \mathrm{wt}}$

15 heterozygous mice were crossed to BAC transgenic Rorc $(\gamma \mathrm{t})$-Cre mice (generated by Gérard Eberl ${ }^{61}$ and

16 provided by Bernhard Ryffel) or CD11c-Cre mice (Itgax-Cre, generated by Boris Reizis ${ }^{62}$ and provided

17 by Véronique Godot). Following intercrossing, co-housed, sex- and age-matched Tmem176a/bl/fl

18 homozygous littermates carrying or not a transgenic Cre allele were used for experiments.

19 OT-I.Ly5.1 homozygous mice were obtained by intercrossing OVA-specific TCR-transgenic OT-I mice

20 (C57BL/6-Tg(TcraTcrb)1100Mjb/Crl) (Charles River) with Ly5.1 mice (B6.SJL-PtprcaPepcb/BoyCrl)

21 (Charles River).

22 OT-II.Ly5.1.Foxp3EGFP homozygous mice were obtained by intercrossing OVA-specific TCR23 transgenic OT-II mice (C57BL/6-Tg(TcraTcrb)425Cbn/Crl) (Charles River) with Ly5.1 mice (B6.SJL- 
1 PtprcaPepcb/BoyCrl) (Charles River) and Foxp3EGFP reporter mice (generated by Bernard

2 Malissen $\left.^{63}\right)$.

3 All mice used for experiments were between 8 and 25 weeks of age and kept under specific pathogen-

4 free conditions. Experimental procedures were carried out in strict accordance with the protocols

5 approved by the Commitee on the Ethics of Animal Experiments of Pays de la Loire and authorized by

6 the French Government's Ministry of Higher Education and Research.

\section{Chemically-induced acute colitis}

8 Mice were given 2\% dextran sulfate sodium (DSS) $(36,000-50,000 \mathrm{MW}$, MP Biomedical) in drinking

9 water ad libitum for 7 days followed by a recovery period without DSS. Mice were monitored and

10 weighed daily.

\section{Citrobacter rodentium infection}

12 Citrobacter rodentium (DBS100, ATCC 51459) were culture aerobically at $37^{\circ} \mathrm{C}$ overnight at $200 \mathrm{rpm}$

13 in Luria-Bertani (LB) broth medium (MP Biomedicals) and then diluted 1:10 in fresh LB medium until

14 the concentration of bacteria reached optical density 600 . Mice were pre-treated with $750 \mathrm{mg} / \mathrm{L}$

15 metronidazole (Sigma) in $2.5 \%$ sucrose drinking water for 4 days as previously described ${ }^{64}$, followed by

163 days with regular drinking water. Mice were then fasted $8 \mathrm{~h}$ before the infection by oral gavage with

$172 \times 10^{9}$ colony-forming units (CFUs) of C. rodentium resuspended in sterile $0.9 \% \mathrm{NaCl}$. Bacterial

18 concentration was assessed via serial dilution on LB agar plates to confirm the CFUs administered. Mice

19 were monitored and weighed daily. Faeces were collected at days 0 and 6 post-infection for detection of

20 C. rodentium by qPCR.

\section{Skin transplantation}


1 Mice were anesthetized with a mixture of 5\% xylazine (Rompun) and $18 \%$ ketamine in PBS (170 $\mu \mathrm{L})$

2 injected intraperitoneally $(8.5 \mathrm{mg} / \mathrm{kg}$ of xylazine and $76.5 \mathrm{mg} / \mathrm{kg}$ of ketamine per mouse). Square skin

3 grafts $\left(1 \mathrm{~cm}^{2}\right)$ were prepared from the tail of male donors and transplanted on the shaved left flank of

4 female recipients. The grafts were fixed to the graft bed with 10-12 interrupted sutures and were covered

5 with protective tape. Mice were monitored every other day and graft rejection was defined as complete

6 sloughing or a dry scab.

\section{$7 \quad$ Tumor growth models}

8 EG7, MCA101-sOVA ${ }^{65}$ (provided by Clotilde Théry) and B16-OVA tumor cells were recovered from

$9 \log$ phase in vitro growth and $1 \times 10^{6}$ cells were injected subcutaneously in $50 \mu \mathrm{L}$ of cold PBS into the

10 flank skin of recipient mice. Tumor growth was measured in a blind fashion with a caliper and expressed

11 as the area based on two perpendicular diameters. Mice were monitored daily and were euthanized when

12 tumor size reached $289 \mathrm{~mm}^{2}$.

\section{Experimental Autoimmune-Encephalomyelitis (EAE)}

14 For EAE induced with MOG peptide, mice were immunized subcutaneously at the base of the tail and

15 lower flanks with $200 \mu \mathrm{g}$ of $\mathrm{MOG}_{35-55}$ peptide (MEVGWYRSPFSRVVHLYRNGK, GenScript) emulsified in complete Freund's adjuvant (Sigma) supplemented with Mycobacterium tuberculosis

17 H37Ra (Difco Laboratories) at $8 \mathrm{mg} / \mathrm{mL}(400 \mu \mathrm{g} / \mathrm{mL}$ per mouse). Pertussis toxin (200 ng, Calbiochem) was injected intraperitoneally on the day of immunization and 2 days later.

19 For EAE induced with MOG protein ${ }^{66}$, mice were immunized subcutaneously at the base of the tail and

20 lower flanks with $500 \mu \mathrm{g}$ of $\mathrm{mMOGTag}$ protein (mouse $\mathrm{MOG}_{1-125}$ extracellular domain fused to a tag 21 for stability and purification purposes) provided by Steven Kerfoot and emulsified in complete Freund's 
1 adjuvant. Pertussis toxin (250 ng) was injected intraperitoneally on the day of immunization and 2 days

2 later.

3 Mice were scored daily for EAE clinical signs on a scale of $0-5: 0$, no disease; 1 , complete limp tail; 2,

$4 \operatorname{limp}$ tail with unilateral hindlimb paralysis; 3, bilateral hindlimb paralysis; 4, bilateral hindlimb paralysis and forelimb weakness (end point). The observer was blinded to the genotype during the scoring.

\section{Delayed-type hypersensitivity (DTH) assay}

7 Mice were immunized subcutaneously at the base of the tail and lower flanks with $50 \mu g$ of whole OVA

8 protein (grade V, Sigma) or OVA $323-339$ (ISQAVHAAHAEINEAGR) peptide (GenScript) emulsified in

9 complete Freund's adjuvant (Sigma). After 7 days, mice were challenged with $250 \mu \mathrm{g}$ of heat-

10 aggregated OVA ( 2 min incubation at $\left.100^{\circ} \mathrm{C}\right)$ injected $(20 \mu \mathrm{L}$, s.c. $)$ in the right hind footpad whereas the

11 left hind footpad received $250 \mu \mathrm{g}$ of heat-aggregated BSA (Sigma) as a control for non-specific

12 inflammation. Footpad thickness was measured prior to, $24 \mathrm{~h}$ and $48 \mathrm{~h}$ after injection with an electronic

13 digital micrometer. The observer was blinded to the genotype during the scoring.

\section{Flow cytometry analysis and cell sorting}

Antibodies and panels used in this study for FACS analysis and cell sorting are listed in Supplemental

Table 1. Red blood cells were lysed with ammonium chloride. Small intestine and colon lamina propria

17 (siLP an cLP) cells were prepared as previously described ${ }^{6}$. Before all stainings, dead cells were marked for exclusion using Fixable Viability Dye eFluor 506 (eBioscience) or DAPI (Thermo Fisher Scientific) followed by Fc receptor blocking using CD16/32 antibody (BD Biosciences). Intracellular stainings were realized using eBioscience Foxp3 / Transcription Factor Staining Buffer Set except for MHC IICLIP peptide staining where cells were fixed using 4\% PFA before permeabilization and staining using a $0.1 \%$ saponine, $1 \%$ BSA solution in PBS. FACS analyses were performed using BD FACS Canto II or a BD LSRFORTESSA X-20 (BD Biosciences) and FlowJo (Treestar) software. For mean Lancien et al. 
1 fluorescence intensity (MFI) analysis, values were ajusted by substracting the basal signal from

2 fluorescence minus one (FMO) staining for each marker. Absolute cell numbers were determined using

3 CytoCount microspheres (Dako, Agilent). Total and surface expression analysis of MHC II, H2-M, H2-

4 O, Ii (invariant chain, CD74) and MHC II-CLIP peptide complex in spleen cDC1 and cDC2 were

5 performed with or without a fixation/permeabilization step, respectively, and by gating on B cells

$6\left(\mathrm{~B} 220^{+} \mathrm{CD} 11 \mathrm{c}^{-}\right), \mathrm{cDC} 1\left(\mathrm{~B} 220^{-} \mathrm{CD} 11 \mathrm{c}^{+} \mathrm{CD} 11 \mathrm{~b}^{-} \mathrm{CD} 8 \alpha^{+}\right)$or $\mathrm{cDC} 2\left(\mathrm{~B} 220^{-} \mathrm{CD} 11 \mathrm{c}^{+} \mathrm{CD} 11 \mathrm{~b}^{+} \mathrm{CD} 8 \alpha^{-}\right) . \mathrm{H} 2-\mathrm{M}$

7 ( $\alpha \beta 2$ dimer) staining was revealed using a FITC-conjugated anti-rat IgG1 antibody. Alexa Fluor 647-

8 conjugated anti-H2-O $\beta$ was provided by Liza Denzin ${ }^{67}$.

9 Cells were FACS-sorted using BD FACSAria II (BD Biosciences). For verification of Cre-induced loxP 10 recombination, $\mathrm{CD} 11 \mathrm{~b}^{-} \mathrm{TCRb}^{+} \mathrm{CD}^{+} \mathrm{CD}^{-} \mathrm{T}$ cells were FACS-sorted from the lamina propria of the

11 small intestine of mice treated as previously described ${ }^{21}$ with $20 \mu \mathrm{g}$ anti-CD3 (145-2C11, kindly

12 provided by J.A. Bluestone), i.p., at days -3 and -1 before sacrifice. CD $11 \mathrm{c}^{\text {high }} \mathrm{MHC} \mathrm{II}^{+} \mathrm{cDCs}$ were

13 FACS-sorted at high purity ( $>98 \%$ ) from the spleen of naive mice after enrichment of CD11 $\mathrm{c}^{+}$cells

14 using a PE-conjugated anti CD11c antibody and magnetic-activated cell sorting (Anti-PE MicroBeads,

15 Miltenyi Biotec). For in vitro functional analysis, intestinal $\mathrm{CD}^{+} \mathrm{CD}^{+} \mathrm{CD}^{+} \mathrm{T}$ cells and $\mathrm{CD}^{-} \mathrm{CD}^{-}$

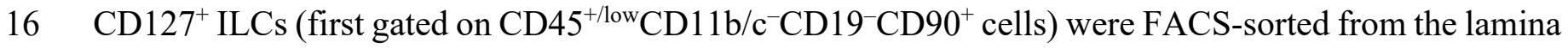

17 propria of the small intestine and colon (pooled). For spleen cDCs used in epigenetic analysis and in

18 vitro culture, $\mathrm{CD} 11 \mathrm{c}^{\text {high }} \mathrm{MHC} \mathrm{II}^{+}$cells were purifed as described above. For RT-qPCR analysis, B cells

$19\left(\mathrm{CD} 19^{+} \mathrm{B} 220^{+} \mathrm{CD} 11 \mathrm{c}^{-}\right), \mathrm{pDCs}\left(\mathrm{CD} 19^{-} \mathrm{B} 220^{+} \mathrm{CD} 11 \mathrm{c}^{+}\right) \mathrm{cDC} 1 \quad\left(\mathrm{CD} 19^{-} \mathrm{B} 220^{-} \mathrm{CD} 11 \mathrm{c}^{\mathrm{high}} \mathrm{CD} 11 \mathrm{~b}^{-} \mathrm{CD} 8 \alpha^{+}\right)$

20 and $\mathrm{cDC} 2\left(\mathrm{CD} 19^{-} \mathrm{B} 220^{-} \mathrm{CD} 11 \mathrm{c}^{\text {high }} \mathrm{CD} 11 \mathrm{~b}^{+} \mathrm{CD} 8 \alpha^{-}\right)$were FACS-sorted from the spleen of WT mice.

21 OVA-specific $\mathrm{CD}^{+} \mathrm{T}$ cells were purified from the spleen of OT-I.Ly5.1 mice using CD8a ${ }^{+} \mathrm{T}$ Cell

22 Isolation Kit II (Miltenyi Biotec). OVA-specific $\mathrm{CD}^{+} \mathrm{EGFP}^{-}$cells $\mathrm{T}$ conventional cells were FACS- 
1 sorted from the spleen of OT-II.Ly5.1.Foxp3EGFP mice after enrichment using CD4 ${ }^{+}$T Cell Isolation

2 Kit (Miltenyi Biotec).

\section{In vivo antigen-specific $T$-cell proliferation assay}

4 OVA-specific naive $\mathrm{CD}^{+} \mathrm{T}$ and $\mathrm{CD}^{+} \mathrm{T}$ conventional cells from OT-I.Ly5.1 and OT-

5 II.Ly5.1.Foxp3EGFP mice, respectively, were labeled with Cell Proliferation Dye (CPD) eFluor 670

6 (eBioscience) and co-injected (i.v.) at a 1:1 ratio (total of $1-2 \times 10^{6}$ cells per mouse). One day later,

7 recipient mice were administered (i.p.) $100 \mu \mathrm{g}$ EndoFit Ovalbumin protein (InvivoGen). After three

8 days, spleens were harvested and proliferation (CPD dilution) of injected cells $\left(\mathrm{CD} 45.1^{+} \mathrm{CD} 8^{+}\right.$or

$9 \quad \mathrm{CD} 45.1^{+} \mathrm{CD} 4^{+}$) was assessed by flow cytometry.

BMDC generation and in vitro antigen-specific T-cell proliferation assay

11 Bone marrow cells were cultured $\left(0.5 \times 10^{6}\right.$ cells per $\left.\mathrm{mL}\right)$ in the presence of $20 \mathrm{ng} / \mathrm{mL}$ GM-CSF (Miltenyi

12 Biotec) in complete RPMI medium. By day 8, the cells, referred to as BMDCs, were harvested and

13 incubated in 96 -well plate $\left(1 \times 10^{4}\right.$ cells per well $)$ with $250 \mu \mathrm{g} / \mathrm{mL}$ EndoFit Ovalbumin protein

14 (InvivoGen) or $10 \mu \mathrm{g} / \mathrm{mL} \mathrm{OVA}_{323-339}$ (ISQAVHAAHAEINEAGR) peptide (GenScript). After $5 \mathrm{~h}$, the

15 cells were washed three times and CPD-labeled $\mathrm{CD}^{+}$Foxp3 ${ }^{-} \mathrm{T}$ cells purified from OT-

16 II.Ly5.1.Foxp3EGFP mice were added and proliferation (CPD dilution) was assessed by flow cytometry

173 days later.

\section{In vitro stimulation and analysis of cDCs and BMDCs}

19 Purified spleen cDCs were plated in 96-well plate at $1 \times 10^{5}$ cells and incubated with or without $0.5 \mu \mathrm{g} / \mathrm{mL}$

20 LPS (Sigma) for $16 \mathrm{~h}$ before the quantification of IL-12p40 and IL-6 in the supernatant by ELISA (BD

21 Biosciences). For phenotypic analysis (MHC II, MHC I, CD80, CD86) by flow cytometry, bulk spleen 
1 cells (comprising $\mathrm{cDC} 1$ and $\mathrm{cDC} 2$ identified using the markers CD11c, CD11b and CD8 $\alpha$ as indicated)

2 or BMDCs were stained freshly of stimulated for $6 \mathrm{~h}$ with $0.5 \mu \mathrm{g} / \mathrm{mL} \mathrm{LPS.}$

\section{In vitro stimulation of $\mathrm{CD4}^{+} \mathrm{T}$ cells and ILCs}

4 Purified intestinal CD4 ${ }^{+} \mathrm{T}$ cells and ILCs were cultured in vitro in 96-well plate (10,000 cells per well

5 in triplicates) in complete medium (Gibco, Thermo Fisher Scientific) for 18 hours in the presence of

6 anti-CD3/CD28 Dynabeads (Thermo Fisher Scientific) at a ratio of 2:1 or IL-23 \pm IL-7, IL-2, IL-1 $\beta$

7 (R\&D Systems) at $50 \mathrm{ng} / \mathrm{mL}$ (except IL-2 at $50 \mathrm{IU} / \mathrm{mL}$ ), respectively. IL-17A, IL-17F, IL-22 (R\&D

8 Systems) and IFN $\gamma$ (BD Biosciences) were then measured in the supernatant by ELISA.

\section{$9 \quad$ In vitro antigen uptake and degradation assays}

10 Antigen endocytosis and degradation in $\mathrm{cDCs}\left(\mathrm{CD} 11 \mathrm{c}^{+} \mathrm{MHCII}^{+}\right)$were assessed by flow cytometry after

11 incubating one million bulk splenocytes in 96 -well plate with $50 \mu \mathrm{g} / \mathrm{mL}$ OVA-FITC or DQ-OVA

12 (Invitrogen, Thermofisher Scientific), respectively. As controls, cells were incubated at $4^{\circ} \mathrm{C}$ or treated with Bafilomycin A (Sigma).

\section{ChIP-seq experiments}

15 Highly purified spleen cDCs $\left(1 \times 10^{6}\right.$ cells from two pooled mice for each preparation $)$ were resuspended in $40 \mu \mathrm{L}$ of PBS. Cells were lysed and chromatin was fragmented with 300 units of Micrococcal nuclease (MNase) (M0247S, New England Biolabs) per well for $10 \mathrm{~min}$ at $37^{\circ} \mathrm{C}$. After full speed centrifugation, supernatants were collected and filled up to $400 \mu \mathrm{L}$. Two $\mu \mathrm{g}$ of anti-H3K27ac (39133,

19 Active Motif) was used for immunoprecipitation overnight at $4^{\circ} \mathrm{C}$. Twenty five $\mu \mathrm{L}$ of G-protein

20 dynabeads (Invitrogen) were added for rotation for $4 \mathrm{~h}$ at $4^{\circ} \mathrm{C}$. Beads were then washed twice with $21200 \mu \mathrm{L}$ wash buffers with increasing salt concentration. ChIP beads were eluted in $50 \mu \mathrm{L}$ of ChIP elution 22 buffer (50 mM Tris-HCl pH 7.5, 10 mM EDTA, 1\% SDS). ChIP and input samples were digested with 
$1250 \mu \mathrm{g} / \mathrm{mL}$ proteinase K (GEXPRK006R, Eurobio) in $50 \mu \mathrm{L}$ TE buffer for $1 \mathrm{~h}$ at $63^{\circ} \mathrm{C}$. ChIP DNA was

2 purified using phenol chlorophorm. Libraries were then prepared according as previously described ${ }^{68}$.

3 Libraries were verified and equimolar pools were sequenced on a NextSeq 500 (75 bp single-end).

\section{ChIP-seq analysis}

5 Single-end reads were mapped to the mm10 genome by the BWA algorithm and reads mapping to non-

canonical and mitochondrial chromosomes were also removed. For each sample, ChIP-seq peaks were

7 detected using DFilter at a $P$-value threshold of $1 \times 10^{-6}$. All samples passed the quality controls (Fraction

8 of reads in peaks [FRiP] $>3 \%$ and non-redundancy fraction $[\mathrm{NRF}]>0.9)$. A set of consensus peaks was

9 then obtained by taking the union of all peaks and counting reads these peaks using Bedtools. To perform

10 Differential Peak Calling, differentially acetylated (DA) peaks were determined using edgeR after a

11 counts per million (cpm) normalization. DA peaks were defined with a Benjamini-Hochberg Q-value

$12 \leq 5 \%$. For heatmap representation, peaks were rlog transformed. To determine gene ontology enrichment

13 in up-regulated peaks the GREAT tool was used.

\section{Quantitative PCR}

15 Quantitative PCR (qPCR) was performed using ViiA 7 Real-Time PCR System and Fast SYBR Green

16 Master Mix reagent (Applied Biosystems, Thermo Fisher Scientific). Primer sequences are listed in

17 Supplemental Table 1).

18 For Citrobacter rodentium bacterial load quantification, genomic DNA (gDNA) from faeces was 19 purified using QIAamp DNA Stool Mini Kit (Qiagen) and the EspB gene was detected using specific 20 primers and normalized with total bacterial gDNA ( $16 S$ gene) using the $2^{-\Delta \Delta \mathrm{Ct}}$ method.

21 For mRNA quantification, total RNA was isolated using RNeasy Mini Kit or Micro Kit (Qiagen). 22 Reverse transcription was performed using M-MLV Reverse Transcriptase and random primers 
1 following manufacturer's instructions (Thermo Fisher Scientific). Gene-specific primers were designed

2 over different exons to prevent amplification of genomic DNA. Gene expression was normalized to

3 glyceraldehyde 3-phosphate dehydrogenase (Gapdh) and expressed in arbitrary units using the $2^{-\Delta \Delta \mathrm{Ct}}$

4 method.

\section{RUSH system}

6 RUSH experiments were realized as previously described by the Perez group ${ }^{35}$. Plasmid constructs are

7 listed in Supplemental Table 1. When indicated, 250 nM MitoTracker Deep Red or 100 nM LysoTracker

8 Deep Red (Thermo Fisher Scientific) were added 30 min before imaging. HeLa cells $\left(1.5 \times 10^{4}\right)$ were

9 seeded into a $\mu$-Slide 8 Well Chamber Slide (Ibidi) and transfected using Lipofectamine LTX Plus

10 Reagent (Thermo Fisher Scientific). After 24 h, the medium was replaced by medium containing $40 \mu \mathrm{M}$

11 of D-biotin (Sigma). The initial time-lapse acquisition characterizing TMEM176B was performed at

12 Institut Curie (Paris, France) with a thermostat controlled chamber using an Eclipse 80i microscope

13 (Nikon) equipped with a spinning disk confocal head (Perkin) and an Ultra897 iXon camera (Andor).

14 Subsequent RUSH analyses were performed at MicroPICell facility (Nantes, France) with a Confocal

15 Nikon A1 RS microscope. To analyze molecule colocalization, we used the plugin Colocalization Studio

16 that contains pixel-based methods that were introduced in section Pixel-Based Methods in the Icy

17 platform. HeLa-CIITA (generated by Philippe Benaroch ${ }^{37}$ ) were used for the analysis of HLA-DR and

18 HLA-DM.

19 Statistical analysis

20 Statistical analyses were performed using Graphpad Prism (La Jolla, CA, USA) using the Mann-Whitney

21 test or, for survival rates, using the Log-rank (Mantel-cox) test. $P$ values $<0.05$ were considered 22 significant. 


\section{Acknowledgments}

2 This work was supported by IHU-Cesti, Nantes Métropole and Région des Pays de la Loire, Paris

3 Scientifiques Régionaux. This work was realized in the context of the Labex IGO program supported by

4 the Agence Nationale de la Recherche (ANR-11-LABX-0016-01). This work was supported by the

5 Fondation pour la Recherche Médicale, grant number ECO20160736078, to Melanie Lancien. We are

6 grateful to Philippe Hulin, Steven Nedellec and Magalie Feyeux from the MicroPICell imagery core

7 facility (Nantes, France) for excellent assistance with confocal microscopy and to Claire Usal, Pierre

8 Pajot and Jean-Marc Merieau for mouse housing and experimental help.

9 Work performed in the F. Perez laboratory was funded by Centre National de la Recherche Scientifique,

10 the Fondation pour la Recherche Medicale (FRM DEQ20120323723), the Labex CellTisPhyBio, and

11 the Agence Nationale de la Recherche (ANR-12-BSV2-0003-01). The laboratory of F. Perez is part of

12 Labex CelTisPhyBio (11-LBX-0038) and Idex Paris Sciences et Lettres (ANR-10-IDEX-0001-02 PSL).

13 The authors acknowledge the Cell and Tissue Imaging Facility (PICT-IBiSA), Institut Curie, a member

14 of the French National Research Infrastructure, France-BioImaging (ANR10-INBS-04).

\section{Author contributions}

C.L., M.L. and M.C.C. designed and supervised the research. M.L., G.Bienvenu, L.G, S.S., E.M., S.R.,

A.Molle, C.F., G.Beriou P.K., S.A.R, V.D.S., G.Boncompain, J.P. and C.L. performed experiments and analyzed data. A.E., C.F., L.B-D and F.C., performed experiments. S.K., G.M. and F.P. contributed to

key research tools and experiment design. A.Moreau., E.C., S.C., F.P., R.J. and J.P. helped with the study design and data interpretation. C.L., M.L., J.P., A.Molle and C.F. wrote the paper. 
bioRxiv preprint doi: https://doi.org/10.1101/851527; this version posted May 1, 2020. The copyright holder for this preprint (which was not certified by peer review) is the author/funder. All rights reserved. No reuse allowed without permission.

\section{Declaration of interests}

2 The authors declare no competing interests. 
3 Figure 1. Generation of Tmem 176a/b double-deficient mice. (A) Graphical topology of mouse

4 TMEM176A and TMEM176B using Protter. The most conserved amino acids between both molecules and across multiple species are highlighted and mainly concentrate within the three first transmembrane domains and in the N-terminal region. (B) Synthetic view of Tmem176a and Tmem176b expression in immune cells summarized from the literature and public databases (Figure S1). Dark red represents the highest expression. (C) Generation of germline Tmem 176a/b double KO (DKO) mice carrying a large deletion using a dual CRISPR-Cas9 strategy. Tmem176a and Tmem176b genes are oriented in an opposite direction in the same genomic locus and share analogous intron-exon organization, with the respective first coding exons only separated by $3.8 \mathrm{~kb}$. Small arrows labeled BR, BR, AF and AR show the position of the primers used for PCR genotyping of the mutant mice. Exons are shown as filled boxes and untranslated regions as shaded boxes. (D) Generation of conditional Tmem176a/b DKO mice. Small arrows labeled bEf, bEr, aEf and aEr show the position of the primers used for PCR genotyping of the mutant mice. (E-F) Verification of cell-specific deletion in Tmem 176a/b DKO conditional mice crossed to Rorc-Cre (E) or CD11c-Cre (F) mice. Relative mRNA expression of the indicated genes quantified by qPCR in purified small intestine (si) $\mathrm{CD}^{+} \mathrm{T}$ cells from mice treated with anti-CD3 and in purified splenic cDCs from naive mice.

Tmem 176a/b double-deficient mice. (A) Relative frequencies of the indicated populations in the small intestine (siLP), colon (cLP) lamina propria and spleen of WT and Tmem176a/b DKO littermate mice. Data shown are means $( \pm \mathrm{SD})$ of three independent experiments. $(B)$ In vitro cytokine secretion by $\mathrm{CD} 4^{+}$ 
$1 \mathrm{~T}$ cells and ILCs FACS-sorted from the intestinal lamina propria of WT and Tmem176a/b DKO

2 littermate mice following in vitro culture $(18 \mathrm{~h})$ with the indicated stimuli. Data shown are means $( \pm \mathrm{SD})$

3 of triplicates representative of three independent experiments. (C) Acute colitis induced with $2 \%$ DSS

4 in drinking water for 7 consecutive days in germline Tmem 176a/b (left panels) and ROR $\gamma \mathrm{t}^{+}$cell-

5 restricted conditional (right panels) DKO mice in comparison to control mice. Data are presented as

6 percent of initial weight $( \pm$ SEM) and survival (weight loss $>20 \%$ ). (D) Infection with $C$. rodentium

7 administered orally $\left(2 \times 10^{9} \mathrm{CFU}\right)$ in germline Tmem $176 a / b$ and ROR $\gamma \mathrm{t}^{+}$cell-restricted conditional DKO

8 mice in comparison to control mice. (E) Quantification of C. rodentium EspB gene by qPCR in the faeces

9 prior infection and 6 days post-infection. Bars indicate means and dots represent individual mice. ND :

10 Not detected.

Figure 3. Phenotypic analysis of cDCs in Tmem176a/b DKO mice. (A) Hematopoietic stem cell and progenitor numbers in the bone marrow of WT and Tmem $176 a / b$ DKO mice analyzed by flow cytometry. Bars indicate means and dots represent individual mice. (B) Gating strategy (left panels) and quantification (right panels) of spleen cDCs and subsets from WT and Tmem176a/b DKO littermate mice by flow cytometry. Bars indicate means and dots represent individual mice. Data were pooled from three independent experiments. (C) Expression of MHC I, MHC II, CD80 and CD86 molecules at the surface of spleen cDC1 and cDC2 from WT and Tmem 176a/b DKO littermate mice. Cells were analyzed by flow cytometry freshly after cell preparation or upon LPS stimulation for $6 \mathrm{~h}$ in vitro. Shown histograms data are representative of three independent experiments. FMO : Fluorescence minus one. (D) IL-12 and IL-6 secretion by purified cDCs purified from the spleen of WT and Tmem 176a/b DKO littermate mice and cultured for $16 \mathrm{~h}$ with or without LPS. Data were pooled from three independent experiments. (E) Heatmap showing unsupervised clustering z-scores of the differentially acetylated 
1 (DA) peaks between WT and DKO spleen cDCs. (F) Volcano plot of the 962 DA peaks (red). Relevant

2 genes linked to MHC class II pathway are highlighted. (G) Genome browser of $C d 74$, hyperacetylated

3 in DKO. (H) Gene ontology of the 445 DA peaks up-regulated in DKO vs WT. (I) Expression of

4 Tmem176a and Tmem176b assessed by RT-qPCR in the indicated populations FACS-sorted from the

5 spleen. Bars indicate means and dots represent individual mice.

Figure 4. Evaluation of $\mathrm{CD8}^{+}$and $\mathrm{CD4}^{+} \mathrm{T}$ cell-dependent models in Tmem 176a/b DKO mice. (A).

9 Rejection rates of male skin graft transplanted onto female WT and Tmem $176 a / b$ DKO mice. Donor and recipient mice were matched for the presence or absence of Tmem176a/b. (B) Survival curves of WT and Tmem176a/b DKO mice injected with EG7, MCA101-sOVA or B16-OVA tumor cell line. (C) Schematic representation of EAE induction using $\mathrm{MOG}_{35-55}$ peptide or $\mathrm{MOG}_{1-125}$ protein. $\mathrm{PT}$ : Pertussis toxin. (D) EAE incidence and score (means \pm SEM) in WT and Tmem176a/b DKO mice using MOG $\mathrm{MS}_{35}$ 55 peptide for immunization. (E) EAE incidence and score (means \pm SEM) in WT and Tmem 176a/b DKO mice using $\mathrm{MOG}_{1-125}$ protein for immunization. (F) Schematic representation of DTH response using OVA $_{323-339}$ peptide or whole OVA protein for immunization. (G) Left (control) and right footpad swelling (means $\pm \mathrm{SEM}$ ) upon injection with the indicated heat-aggregated proteins in WT and

Tmem 176a/b DKO mice using OVA $323-339$ peptide for immunization. (H) Left (control) and right footpad swelling (means \pm SEM) upon injection with the indicated heat-aggregated proteins in WT and Tmem176a/b DKO mice using whole OVA protein for immunization. 
tracking the injected OVA-specific T cells by flow cytometry. CPD : cell proliferation dye. (B)

2 Representative histograms showing the level of proliferation (reflected by CPD dilution) of the OVA3 specific $\mathrm{CD}^{+}$and $\mathrm{CD}^{+} \mathrm{T}$ cells following OVA protein injection in WT and Tmem176a/b DKO mice. (C) Quantification of OVA-specific $\mathrm{CD}^{+}$and $\mathrm{CD}^{+} \mathrm{T}$ cell proliferation in WT and Tmem176a/b DKO mice. (D-E) Quantification of OVA-specific CD4 ${ }^{+} \mathrm{T}$ cell proliferation in ROR $\gamma \mathrm{t}^{+}$cell-restricted (D) or CD11 $\mathrm{c}^{+}$cell-restricted $(\mathbf{E})$ conditional DKO mice. Bars indicate means and dots represent individual

mice. Data were pooled from at least two independent experiments. (F) Schematic representation of the in vitro antigen-specific $\mathrm{CD}^{+} \mathrm{T}$ cell proliferation assay using BMDCs as antigen-presenting cells. (G) Quantification of $\mathrm{CD}^{+} \mathrm{T}$ cell proliferation following incubation of BMDCs from WT and Tmem176a/b DKO mice with $\mathrm{OVA}_{323-339}$ peptide or whole OVA protein. Data shown are means $( \pm \mathrm{SD})$ of triplicates and are representative of two independent experiments.

DCs. (A) Schematic overview of the MHC II pathway. The late endocytic compartment in which prevents premature loading with endogenous peptides before HLA-DM (H2-M in mouse) catalyzes the release of the class II-associated invariant chain peptide (CLIP) in the MIIC. Another MHC II-like protein, HLA-DO (H2-O in mouse), may add another level of regulation by inhibiting H2-M. ILVs : intraluminal vesicles. (B) In vitro antigen uptake and degradation by spleen cDCs from WT and 
and MHC II-CLIP complex molecules in B cells, cDC1 and cDC2 populations from the spleen of WT and Tmem 176a/b DKO mice. Bars indicate means and dots represent individual mice. Data were pooled from two independent experiments.

Figure 7. Intracellular trafficking of TMEM176A and TMEM176B using the RUSH system. (A)

7 Principles of the retention using selective hooks (RUSH) system. In the setting we chose to implement for this two-state assay, the protein of interest is fused to the streptavidin binding protein (SBP) and is retained in the donor compartment (here the endoplasmic reticulum, ER) in which the hook (here an isoform of the invariant chain fused to the core streptavidin) remains localized. Synchronous release of the protein of interest is induced by addition of biotin and intracellular trafficking can be monitored by measuring fluorescent tags such as enhanced (E)GFP or mCherry signal by time-lapse confocal microscopy. (B) Schematic representation of the different intracellular compartments and associated markers analyzed. Proteins analyzed using RUSH constructs are indicated (C) Micrographs of HeLa cells expressing the TMEM176B-EGFP RUSH construct prior and after addition of biotin. Bar, $10 \mu \mathrm{m}$. (D) Dual-color analysis using the TMEM176A-EGFP and TMEM176B-mCherry RUSH constructs. Insets show higher magnifications of regions of interest. Circles show examples of colocalized signals. Dual-color analysis using the TMEM176B-mCherry or TMEM176B-EGFP RUSH constructs and the indicated genes or probes, $>40$ min after biotin addition. (H-I) Dual-color analysis in Hela-CIITA cells using the TMEM176B-mCherry RUSH construct with the YFP-associated HLA-DR (H) or HLA-DM (I) plasmids, $>40$ min after biotin addition. To avoid interference of the ER-resident mutated form of Ii

24 (in the TMEM176B RUSH construct) on normal dynamic of the MHC II pathway, Ii was replaced by 
1 Golgin-84 as a hook in a new construct. To compensate for the low transfection efficiency of the DR/DM

2 constructs, $\mathrm{YFP}^{+}$cells were FACS-sorting $24 \mathrm{~h}$ before imaging. (J) Pearson's correlation coefficients

3 comparing TMEM176B with CD44, HLA-DR and HLA-DM signals. Each dot represents an individual

4 cell. 
List of Supplemental data

2

$3 \quad$ Figure S1. Tmem176a and Tmem176b expression in the immune system.

4

5 Figure S2. Gating strategy for T cell and ILC FACS analysis and gene expression analysis by RT-

6 qPCR analysis.

8 Figure S3. Gating strategy for bone-marrow progenitor analysis by flow cytometry.

10 Table S1. List of antibodies, oligonucleotides and plasmids used in this study.

Video S1. Real-time imaging of the synchronized trafficking of TMEM176B using the RUSH system (corresponds to Figure 7C).

Video S2. Dual color, real-time imaging of the synchronized trafficking of TMEM176B and

TMEM176A using the RUSH system (corresponds to Figure 7D).

Video S3. Dual color, real-time imaging of the synchronized trafficking of TMEM176B and

TMEM176A using the RUSH system (corresponds to Figure 7E). 


\section{References}

1. Feske, S., Wulff, H. \& Skolnik, E. Y. Ion Channels in Innate and Adaptive Immunity. Annu. Rev. Immunol. 33, 291-353 (2015).

2. Vaeth, M. et al. $\mathrm{Ca}^{2+}$ Signaling but Not Store-Operated $\mathrm{Ca}^{2+}$ Entry Is Required for the Function of Macrophages and Dendritic Cells. J. Immunol. 195, 1202-1217 (2015).

3. Nunes-Hasler, P. et al. STIM1 promotes migration, phagosomal maturation and antigen crosspresentation in dendritic cells. Nat. Commun. 8, 1852 (2017).

4. Vandier, C. \& Velge-Roussel, F. Regulation of human dendritic cell immune functions by ion channels. Curr. Opin. Immunol. 52, 27-31 (2018).

5. Segovia, M. et al. Autologous Dendritic Cells Prolong Allograft Survival Through Tmem176b Dependent Antigen Cross-Presentation: Immunoregulatory Mechanisms of Autologous DCs. Am. J. Transplant. 14, 1021-1031 (2014).

6. Drujont, L. et al. ROR $\gamma \mathrm{t}+$ cells selectively express redundant cation channels linked to the Golgi apparatus. Sci. Rep. 6, 23682 (2016).

7. Nickles, D. et al. Blood RNA profiling in a large cohort of multiple sclerosis patients and healthy controls. Hum. Mol. Genet. 22, 4194-4205 (2013).

8. de Jong, K. et al. Genome-wide interaction study of gene-by-occupational exposure and effects on FEV 1 levels. J. Allergy Clin. Immunol. 136, 1664-1672.e14 (2015).

9. Grunin, M., Hagbi-Levi, S.-, Rinsky, B., Smith, Y. \& Chowers, I. Transcriptome Analysis on Monocytes from Patients with Neovascular Age-Related Macular Degeneration. Sci. Rep. 6, 29046 (2016).

10. Louvet, C. et al. Identification of a New Member of the CD20/FcepsilonRIbeta Family Overexpressed in Tolerated Allografts. Am. J. Transplant. 5, 2143-2153 (2005).

11. Song, C. et al. Unique and redundant functions of $\mathrm{NKp} 46^{+}$ILC3s in models of intestinal inflammation. J. Exp. Med. 212, 1869-1882 (2015).

12. Engel, I. et al. Innate-like functions of natural killer T cell subsets result from highly divergent gene programs. Nat. Immunol. 17, 728-739 (2016).

13. Kohlgruber, A. C. et al. $\gamma \delta \mathrm{T}$ cells producing interleukin-17A regulate adipose regulatory $\mathrm{T}$ cell homeostasis and thermogenesis. Nat. Immunol. 19, 464-474 (2018).

14. Tan, L. et al. Single-Cell Transcriptomics Identifies the Adaptation of Scart1+ V $\gamma 6+\mathrm{T}$ Cells to Skin Residency as Activated Effector Cells. Cell Rep. 27, 3657-3671.e4 (2019).

15. Ciofani, M. et al. A Validated Regulatory Network for Th17 Cell Specification. Cell 151, 289-

Lancien et al. 
303 (2012).

16. Zuccolo, J. et al. Phylogenetic Analysis of the MS4A and TMEM176 Gene Families. PLoS ONE 5, e9369 (2010).

17. Condamine, T. et al. Tmem176B and Tmem176A are associated with the immature state of dendritic cells. J. Leukoc. Biol. 88, 507-515 (2010).

18. Vandenbon, A. et al. Immuno-Navigator, a batch-corrected coexpression database, reveals cell type-specific gene networks in the immune system. Proc. Natl. Acad. Sci. U. S. A. 113, E23932402 (2016).

19. The Immunological Genome Project Consortium et al. The Immunological Genome Project: networks of gene expression in immune cells. Nat. Immunol. 9, 1091-1094 (2008).

20. Lemoine, A., Chauveau-Le Friec, G., Langa, F. \& Louvet, C. Generation of a Double KO Mouse by Simultaneous Targeting of the Neighboring Genes Tmem176a and Tmem176b Using CRISPR/Cas9: Key Steps from Design to Genotyping. J. Genet. Genomics 43, 329-340 (2016).

21. Esplugues, E. et al. Control of TH17 cells occurs in the small intestine. Nature 475, 514-518 (2011).

22. Brown, C. C. et al. Transcriptional Basis of Mouse and Human Dendritic Cell Heterogeneity. Cell 179, 846-863.e24 (2019).

23. Eberl, G. ROR $\gamma t$, a multitask nuclear receptor at mucosal surfaces. Mucosal Immunol. 10, 27-34 (2017).

24. Sugimoto, K. et al. IL-22 ameliorates intestinal inflammation in a mouse model of ulcerative colitis. J. Clin. Invest. 118, 534-544 (2008).

25. Zenewicz, L. A. et al. Innate and Adaptive Interleukin-22 Protects Mice from Inflammatory Bowel Disease. Immunity 29, 947-957 (2008).

26. Zheng, Y. et al. Interleukin-22 mediates early host defense against attaching and effacing bacterial pathogens. Nat. Med. 14, 282-289 (2008).

27. Rankin, L. C. et al. Complementarity and redundancy of IL-22-producing innate lymphoid cells. Nat. Immunol. 17, 179-186 (2016).

28. Pace, L. et al. The epigenetic control of stemness in $\mathrm{CD}^{+} \mathrm{T}$ cell fate commitment. Science 359, 177-186 (2018).

29. Saeed, S. et al. Epigenetic programming of monocyte-to-macrophage differentiation and trained innate immunity. Science 345, 1251086-1251086 (2014).

30. Dudziak, D. et al. Differential Antigen Processing by Dendritic Cell Subsets in Vivo. Science 315, 
107-111 (2007).

31. Segovia, M. et al. Targeting TMEM176B Enhances Antitumor Immunity and Augments the Efficacy of Immune Checkpoint Blockers by Unleashing Inflammasome Activation. Cancer Cell 35, 767-781.e6 (2019).

32. Meredith, M. M. et al. Expression of the zinc finger transcription factor zDC (Zbtb46, Btbd4) defines the classical dendritic cell lineage. J. Exp. Med. 209, 1153-1165 (2012).

33. ten Broeke, T., Wubbolts, R. \& Stoorvogel, W. MHC Class II Antigen Presentation by Dendritic Cells Regulated through Endosomal Sorting. Cold Spring Harb. Perspect. Biol. 5, a016873a016873 (2013).

34. Shui, W. et al. Membrane proteomics of phagosomes suggests a connection to autophagy. Proc. Natl. Acad. Sci. 105, 16952-16957 (2008).

35. Boncompain, G. et al. Synchronization of secretory protein traffic in populations of cells. Nat. Methods 9, 493-498 (2012).

36. Chen, Y., Gershlick, D. C., Park, S. Y. \& Bonifacino, J. S. Segregation in the Golgi complex precedes export of endolysosomal proteins in distinct transport carriers. J. Cell Biol. 216, 41414151 (2017).

37. Stumptner-Cuvelette, P. et al. HIV-1 Nef impairs MHC class II antigen presentation and surface expression. Proc. Natl. Acad. Sci. 98, 12144-12149 (2001).

38. Zwart, W. et al. Spatial Separation of HLA-DM/HLA-DR Interactions within MIIC and Phagosome-Induced Immune Escape. Immunity 22, 221-233 (2005).

39. Lurton, J., Rose, T. M., Raghu, G. \& Narayanan, A. S. Isolation of a Gene Product Expressed by a Subpopulation of Human Lung Fibroblasts by Differential Display. Am. J. Respir. Cell Mol. Biol. 20, 327-331 (1999).

40. Maeda, Y. et al. Role of Clast1 in development of cerebellar granule cells. Brain Res. 1104, 18-26 (2006).

41. Kang, S. et al. Identification of nuclear hormone receptor pathways causing insulin resistance by transcriptional and epigenomic analysis. Nat. Cell Biol. 17, 44-56 (2015).

42. Otsubo, T. et al. Identification of novel targets for antiangiogenic therapy by comparing the gene expressions of tumor and normal endothelial cells. Cancer Sci. 105, 560-567 (2014).

43. Gao, D. et al. Methylation of TMEM176A is an independent prognostic marker and is involved in human colorectal cancer development. Epigenetics 12, 575-583 (2017).

44. Ryu, S.-H. et al. Oncogenic Ras-mediated downregulation of Clast1/LR8 is involved in Rasmediated neoplastic transformation and tumorigenesis in NIH3T3 cells. Cancer Sci. 101, 1990- 
1996 (2010).

45. Vanden Berghe, T. et al. Passenger Mutations Confound Interpretation of All Genetically Modified Congenic Mice. Immunity 43, 200-209 (2015).

46. Chisolm, D. A. et al. Defining Genetic Variation in Widely Used Congenic and Backcrossed Mouse Models Reveals Varied Regulation of Genes Important for Immune Responses. Immunity 51, 155-168.e5 (2019).

47. Hepworth, M. R. et al. Innate lymphoid cells regulate CD4+ T-cell responses to intestinal commensal bacteria. Nature 498, 113-117 (2013).

48. Hepworth, M. R. et al. Group 3 innate lymphoid cells mediate intestinal selection of commensal bacteria-specific CD4+ T cells. Science 348, 1031-1035 (2015).

49. Dikiy, S. \& Rudensky, A. Y. Mouse Watch: A Cautionary Tale. Immunity 51, 10-12 (2019).

50. Ghosh, H. S., Cisse, B., Bunin, A., Lewis, K. L. \& Reizis, B. Continuous Expression of the Transcription Factor E2-2 Maintains the Cell Fate of Mature Plasmacytoid Dendritic Cells. Immunity 33, 905-916 (2010).

51. Jaitin, D. A. et al. Massively Parallel Single-Cell RNA-Seq for Marker-Free Decomposition of Tissues into Cell Types. Science 343, 776-779 (2014).

52. Villani, A.-C. et al. Single-cell RNA-seq reveals new types of human blood dendritic cells, monocytes, and progenitors. Science 356, eaah4573 (2017).

53. Binnewies, M. et al. Unleashing Type-2 Dendritic Cells to Drive Protective Antitumor CD4+ T Cell Immunity. Cell 177, 556-571.e16 (2019).

54. Merad, M., Sathe, P., Helft, J., Miller, J. \& Mortha, A. The Dendritic Cell Lineage: Ontogeny and Function of Dendritic Cells and Their Subsets in the Steady State and the Inflamed Setting. Annu. Rev. Immunol. 31, 563-604 (2013).

55. Gerner, M. Y., Casey, K. A., Kastenmuller, W. \& Germain, R. N. Dendritic cell and antigen dispersal landscapes regulate T cell immunity. J. Exp. Med. 214, 3105-3122 (2017).

56. Casey, J. R., Grinstein, S. \& Orlowski, J. Sensors and regulators of intracellular pH. Nat. Rev. Mol. Cell Biol. 11, 50-61 (2010).

57. van den Hoorn, T., Paul, P., Janssen, L., Janssen, H. \& Neefjes, J. Dynamics within tetraspanin pairs affect MHC class II expression. J. Cell Sci. 125, 328-339 (2012).

58. Global Lipids Genetics Consortium. Discovery and refinement of loci associated with lipid levels. Nat. Genet. 45, 1274-1283 (2013).

59. Kuipers, H. F. et al. Statins Affect Cell-Surface Expression of Major Histocompatibility Complex 
60. Birling, M.-C., Dierich, A., Jacquot, S., Hérault, Y. \& Pavlovic, G. Highly-efficient, fluorescent, locus directed cre and FlpO deleter mice on a pure C57BL/6N genetic background. genesis 50, 482-489 (2012).

61. Lochner, M. et al. In vivo equilibrium of proinflammatory $\mathrm{IL}-17^{+}$and regulatory $\mathrm{IL}-10^{+}$Foxp $3^{+}$ ROR $\gamma \mathrm{t}^{+}$T cells. J. Exp. Med. 205, 1381-1393 (2008).

62. Caton, M. L., Smith-Raska, M. R. \& Reizis, B. Notch-RBP-J signaling controls the homeostasis of CD8 ${ }^{-}$dendritic cells in the spleen. J. Exp. Med. 204, 1653-1664 (2007).

63. Wang, Y. et al. Th2 Lymphoproliferative Disorder of Lat ${ }^{Y 136 F}$ Mutant Mice Unfolds Independently of TCR-MHC Engagement and Is Insensitive to the Action of Foxp $3{ }^{+}$Regulatory T Cells. J. Immunol. 180, 1565-1575 (2008).

64. Wlodarska, M. et al. Antibiotic Treatment Alters the Colonic Mucus Layer and Predisposes the Host to Exacerbated Citrobacter rodentium -Induced Colitis. Infect. Immun. 79, 1536-1545 (2011).

65. Zeelenberg, I. S. et al. Targeting Tumor Antigens to Secreted Membrane Vesicles In vivo Induces Efficient Antitumor Immune Responses. Cancer Res. 68, 1228-1235 (2008).

66. Jain, R. W., Dang, A. K. \& Kerfoot, S. M. Simple and Efficient Production and Purification of Mouse Myelin Oligodendrocyte Glycoprotein for Experimental Autoimmune Encephalomyelitis Studies. J. Vis. Exp. 54727 (2016).

67. Fallas, J. L., Yi, W., Draghi, N. A., O’Rourke, H. M. \& Denzin, L. K. Expression Patterns of H2$\mathrm{O}$ in Mouse B Cells and Dendritic Cells Correlate with Cell Function. J. Immunol. 178, 14881497 (2007).

68. Sun, W. et al. Histone Acetylome-wide Association Study of Autism Spectrum Disorder. Cell 167, 1385-1397.e11 (2016). 665 (2005). 


\section{Figure 1}

A

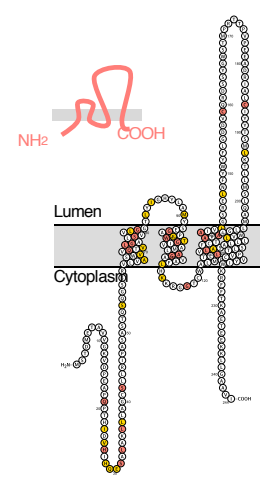

Amino acid conservation between TMEM176A and TMEM176B $\mid \odot$ Complete identity mouse / rat / dog / pig / cattle / chimpanzee / human

C Germline DKO mouse : Tmem $176 a / b^{-1-}$
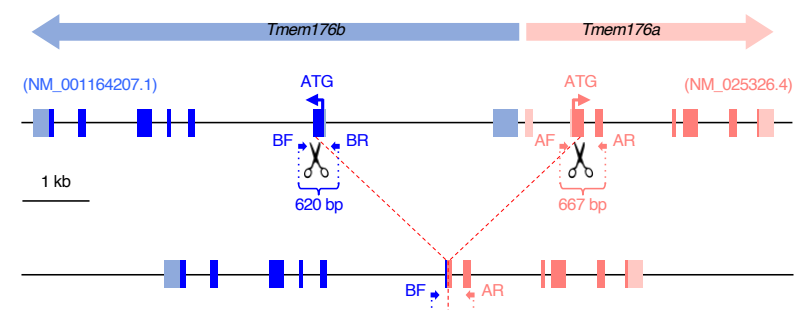

$\underbrace{}_{572 \mathrm{bp}}$
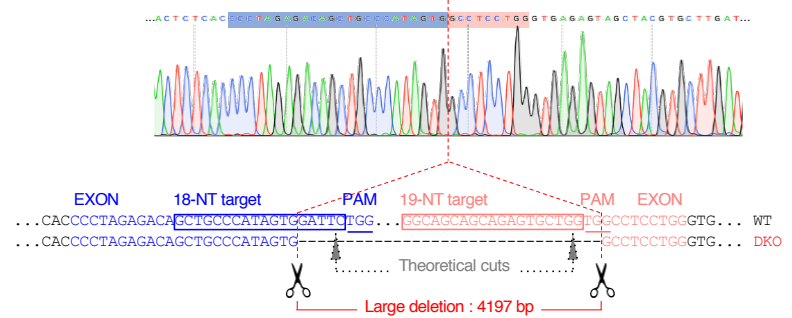

$\Xi$

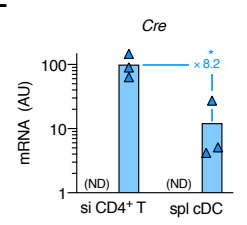

TMEM176B

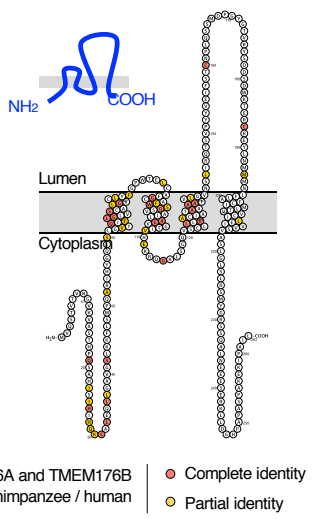

B
Highest expression of Tmem176a and Tmem176b in the immune system

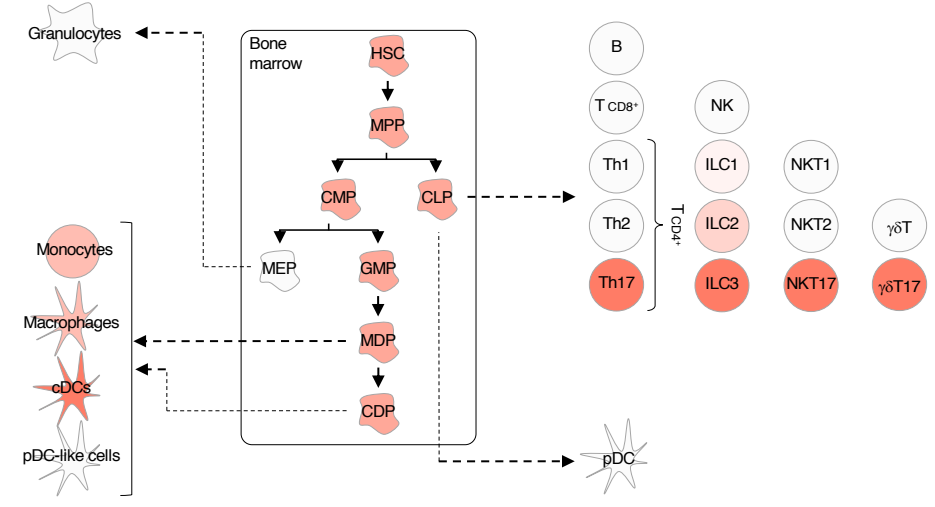

$\mathrm{D}$ Conditional («floxed ») DKO mouse : Tmem176a/b/ffl

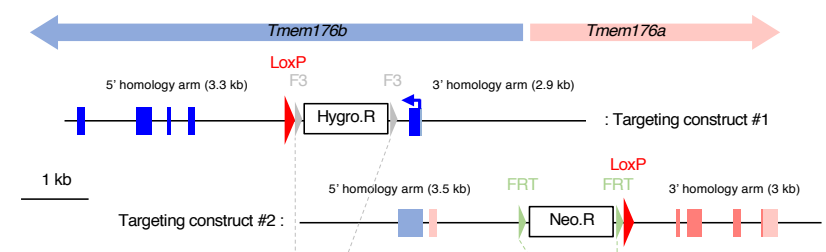

Conditional allele :

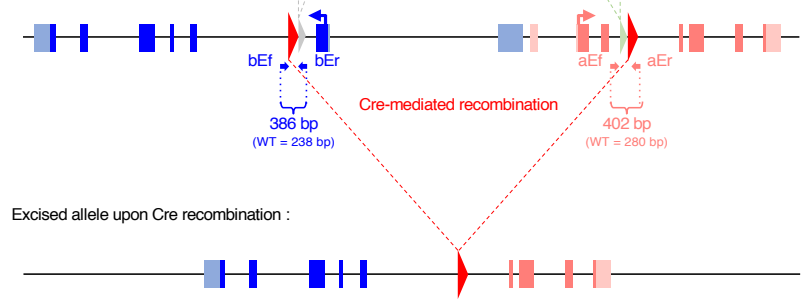

$\mathrm{F}$
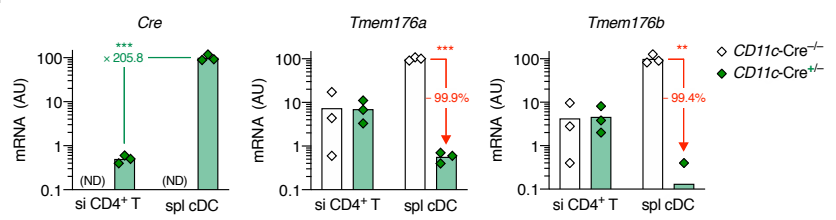


\section{Figure 2}
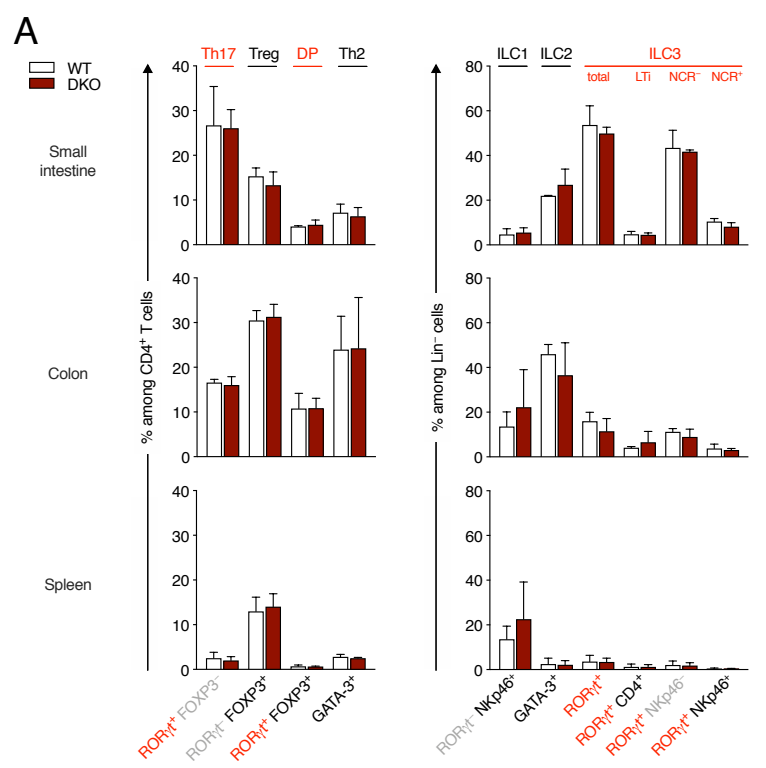

B
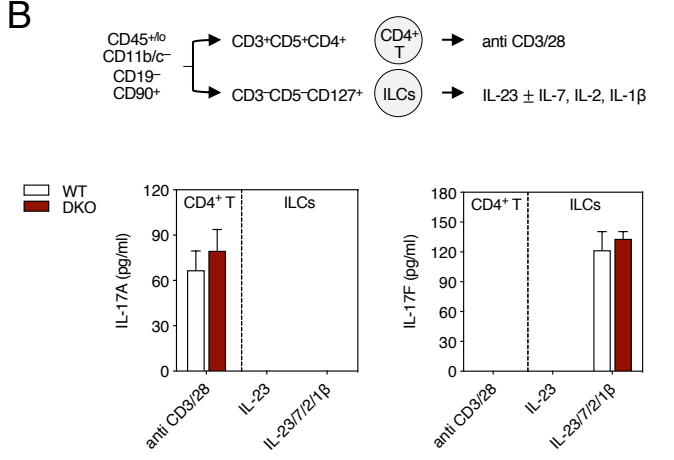

C Germline DKO mice
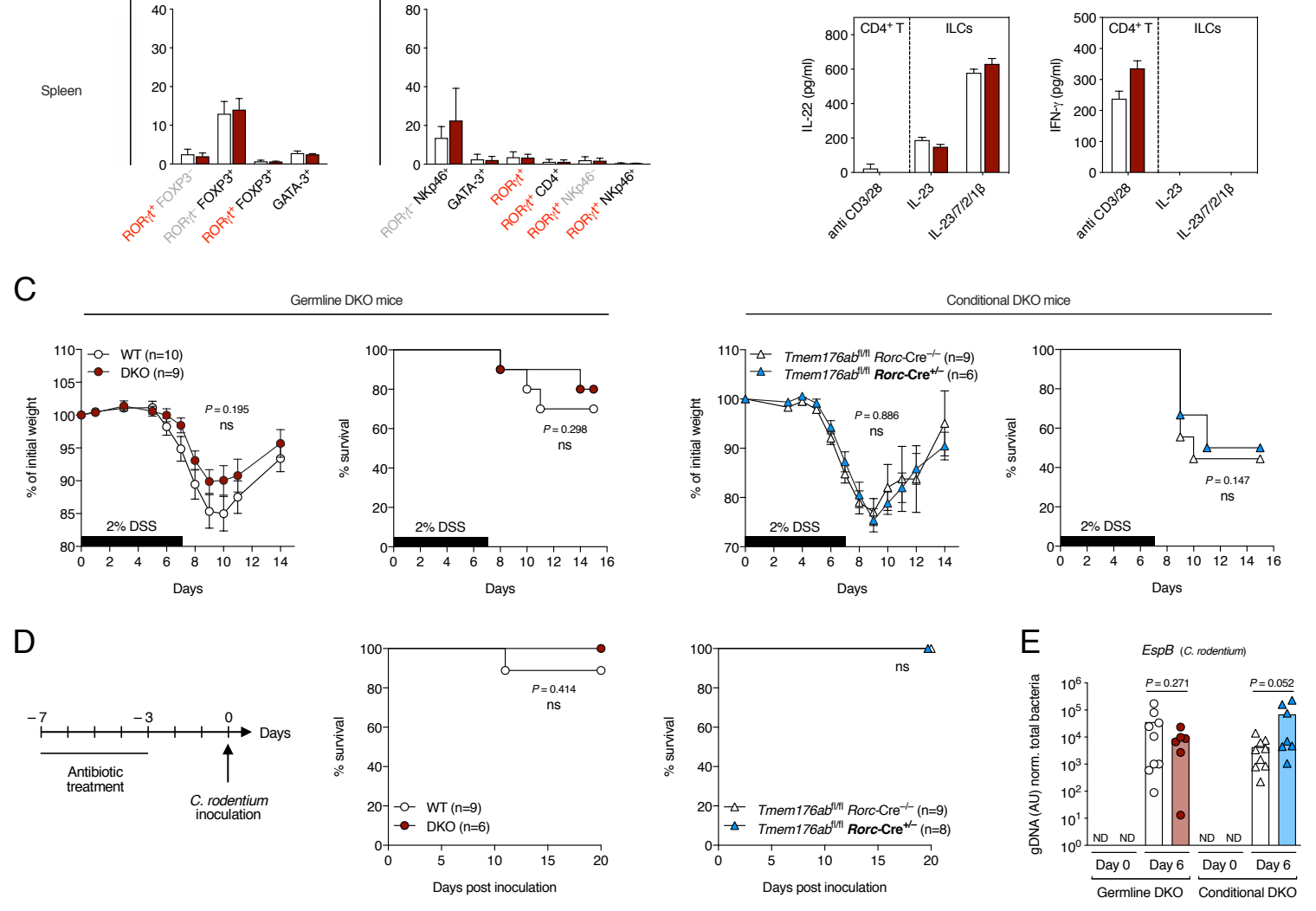
bioRxiv preprint doi: https://doi.org/10.1101/851527; this version posted May 1,2020 . The copyright holder for this preprint (which was not certified by peer review) is the author/funder. All rights reserved. No reuse allowed without permission.

Figure 3

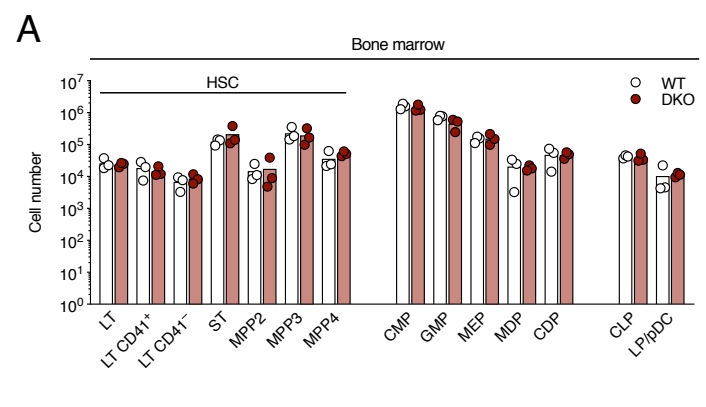

B
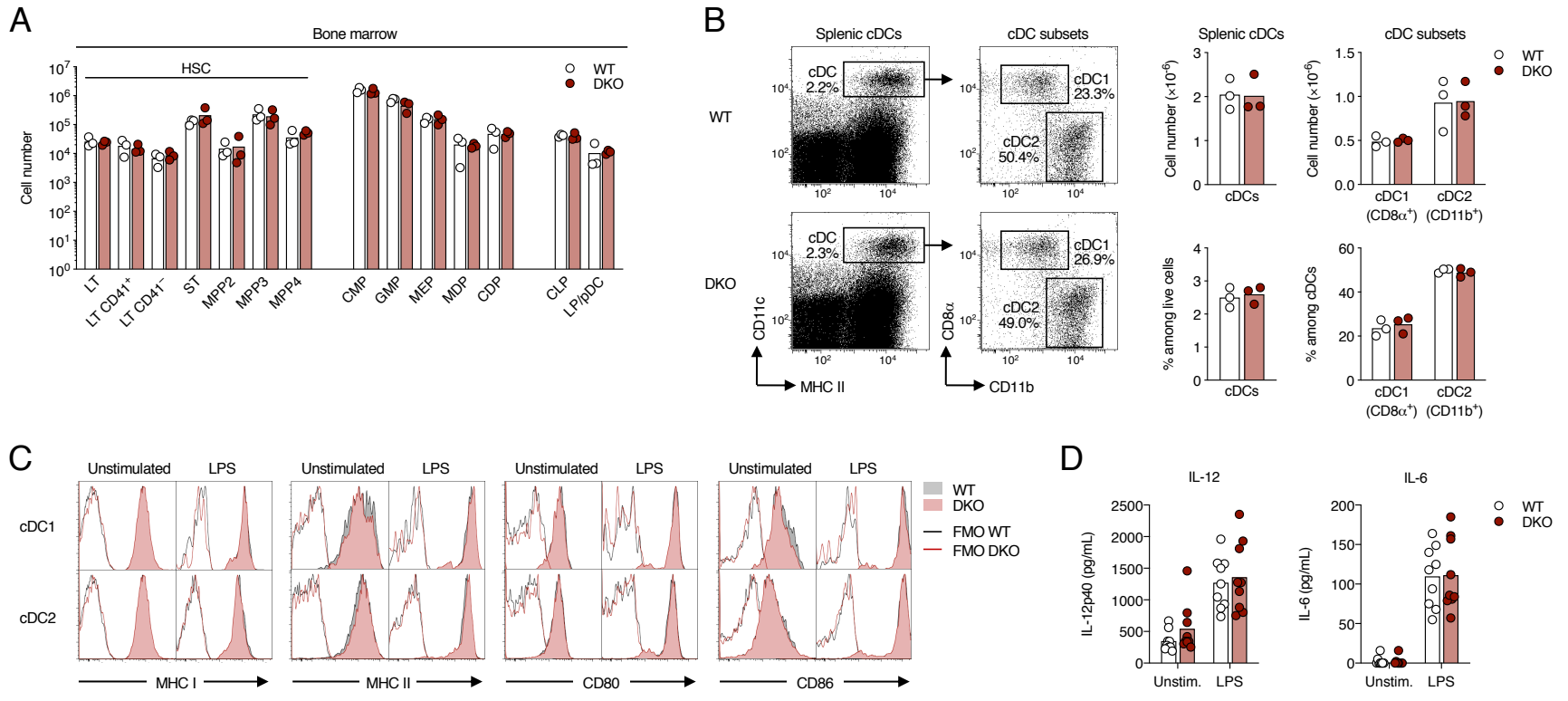

D
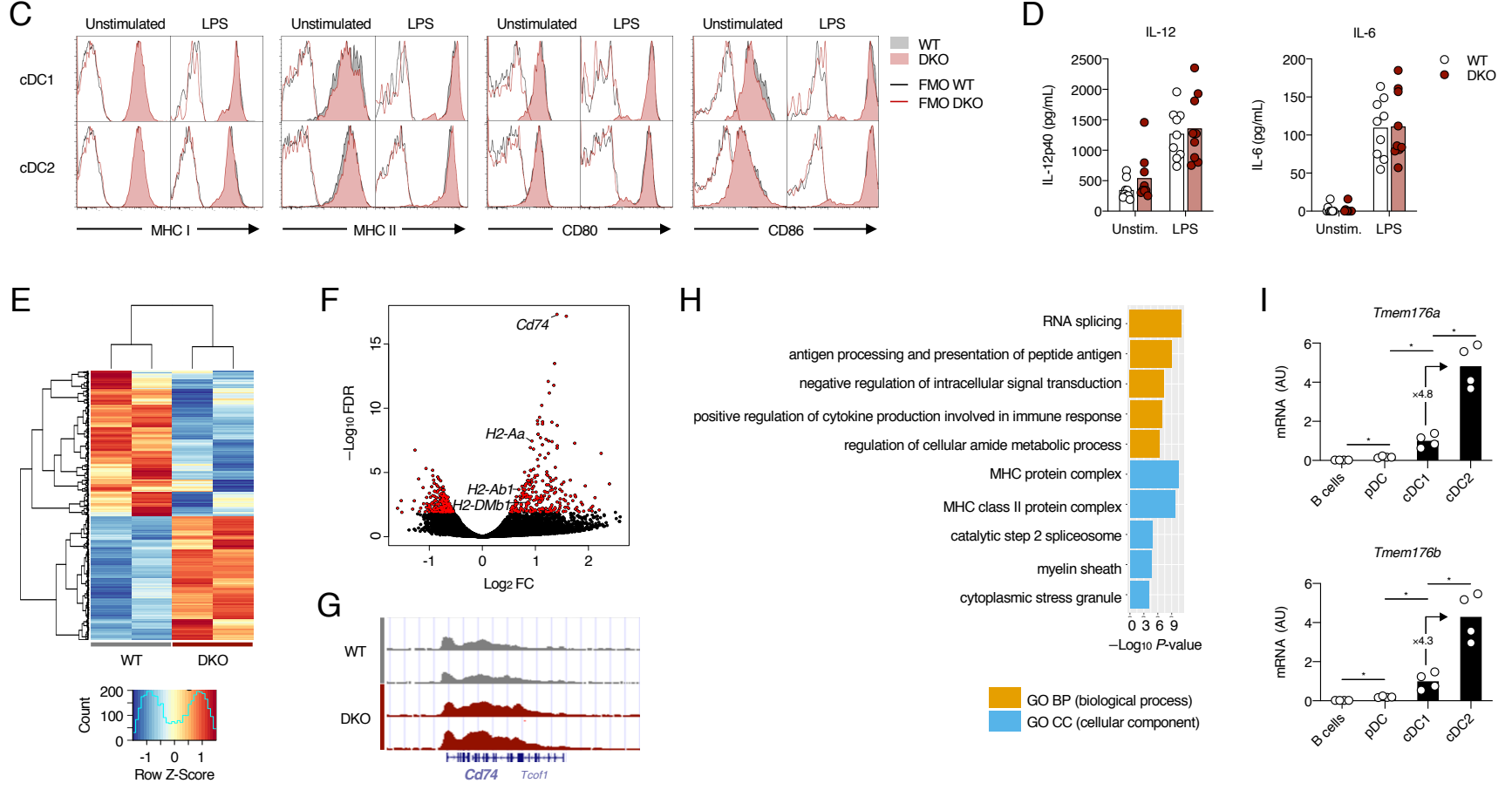


\section{Figure 4}

A

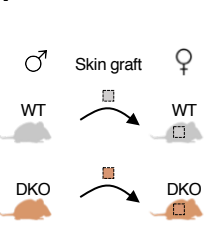

C

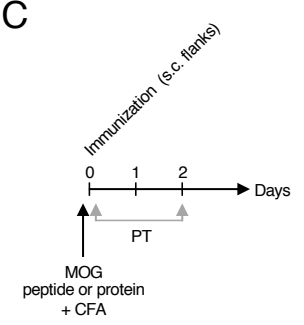

F

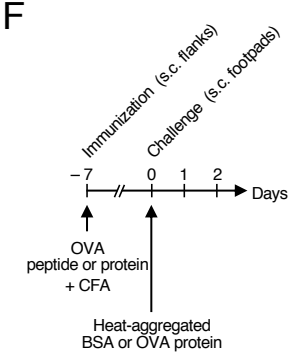

B

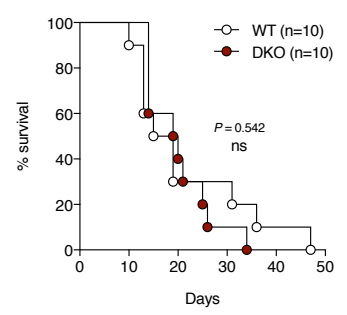

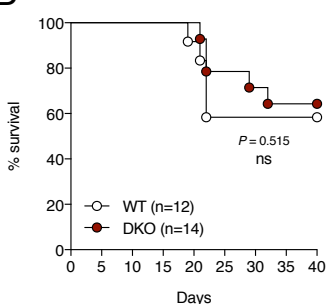
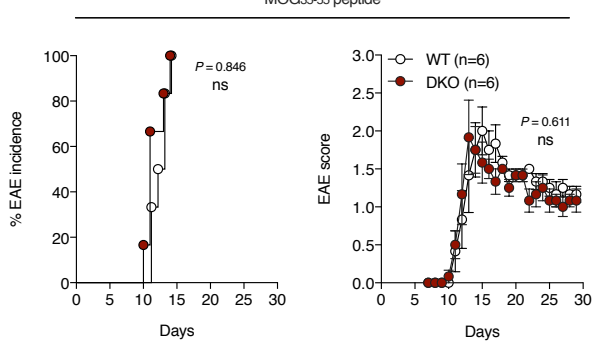
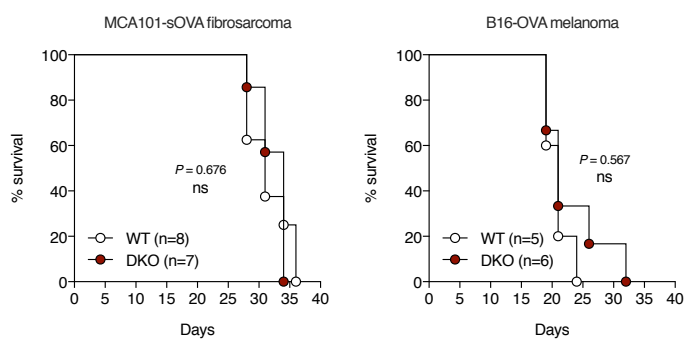

E

MOG protein
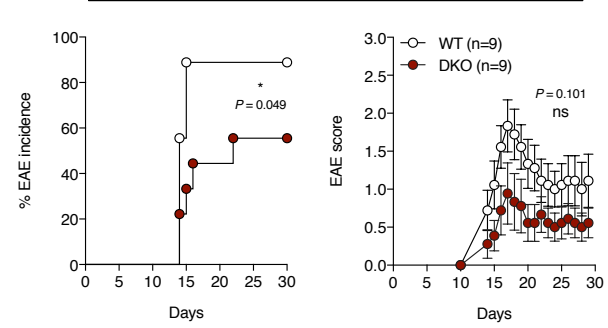

G

Immunization : OVA peptide
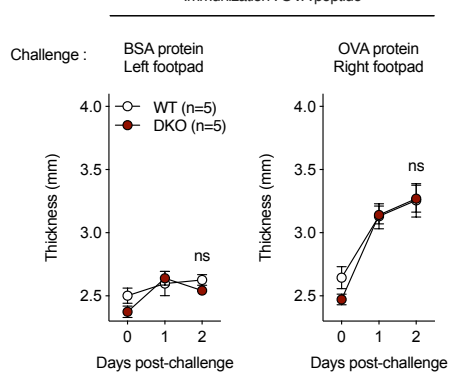

$\mathrm{H}$

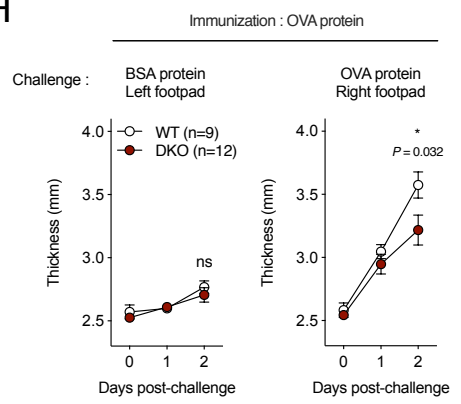




\section{Figure 5}
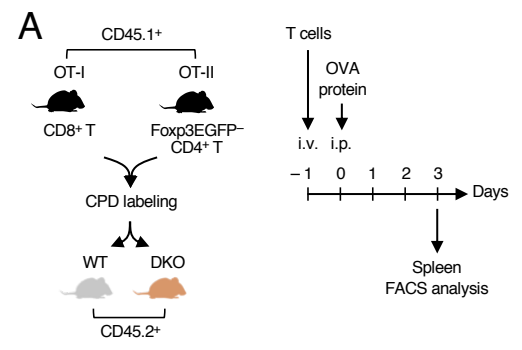

C
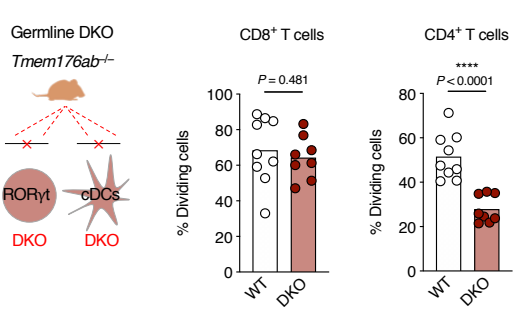

$\mathrm{F}$

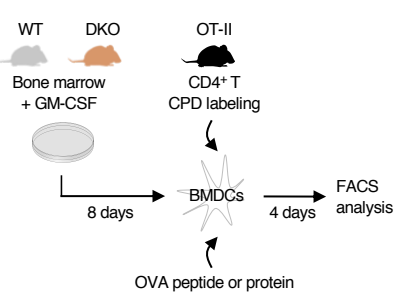

G

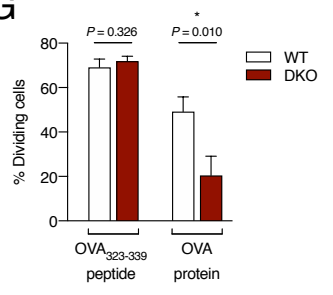

D
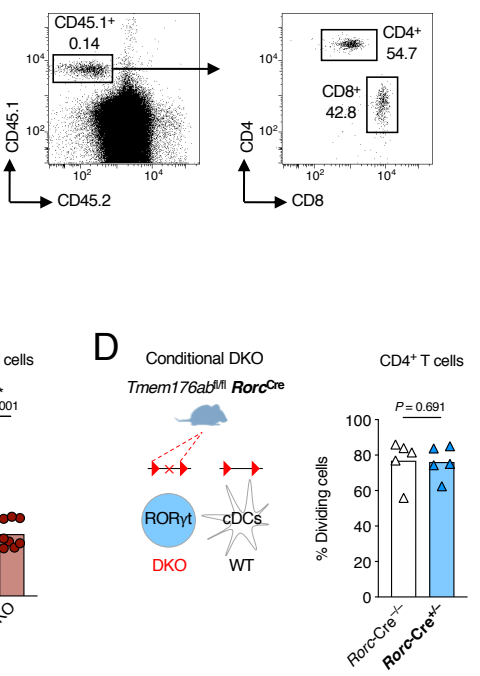

B

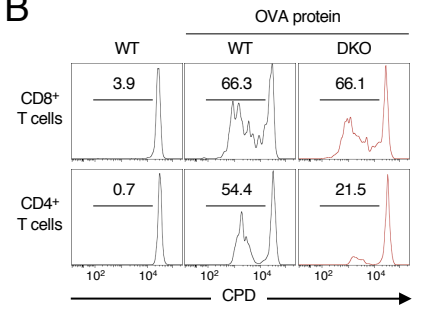

E

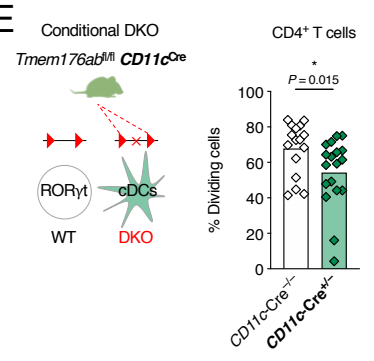


Figure 6
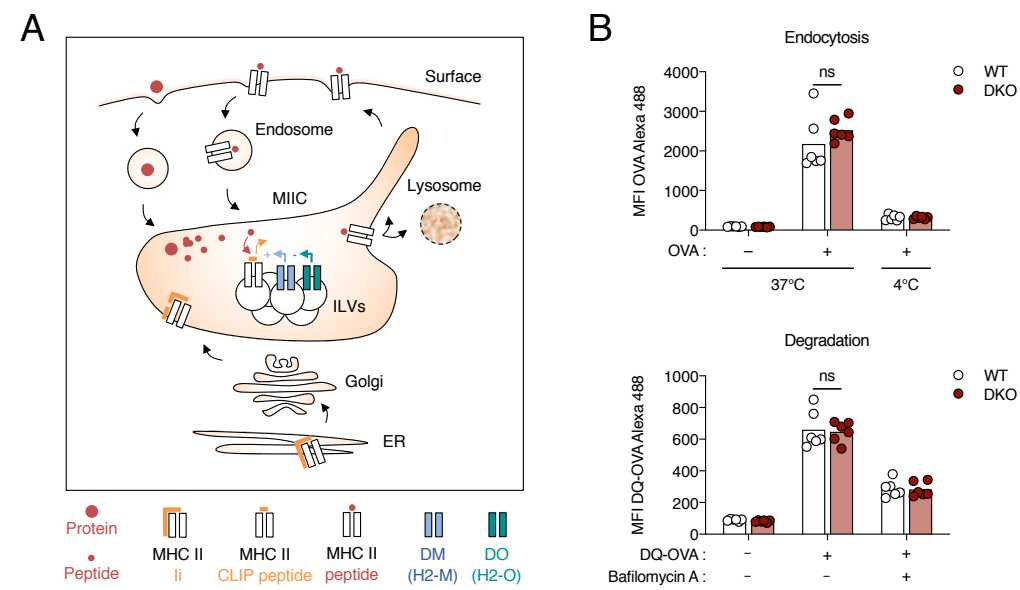

C
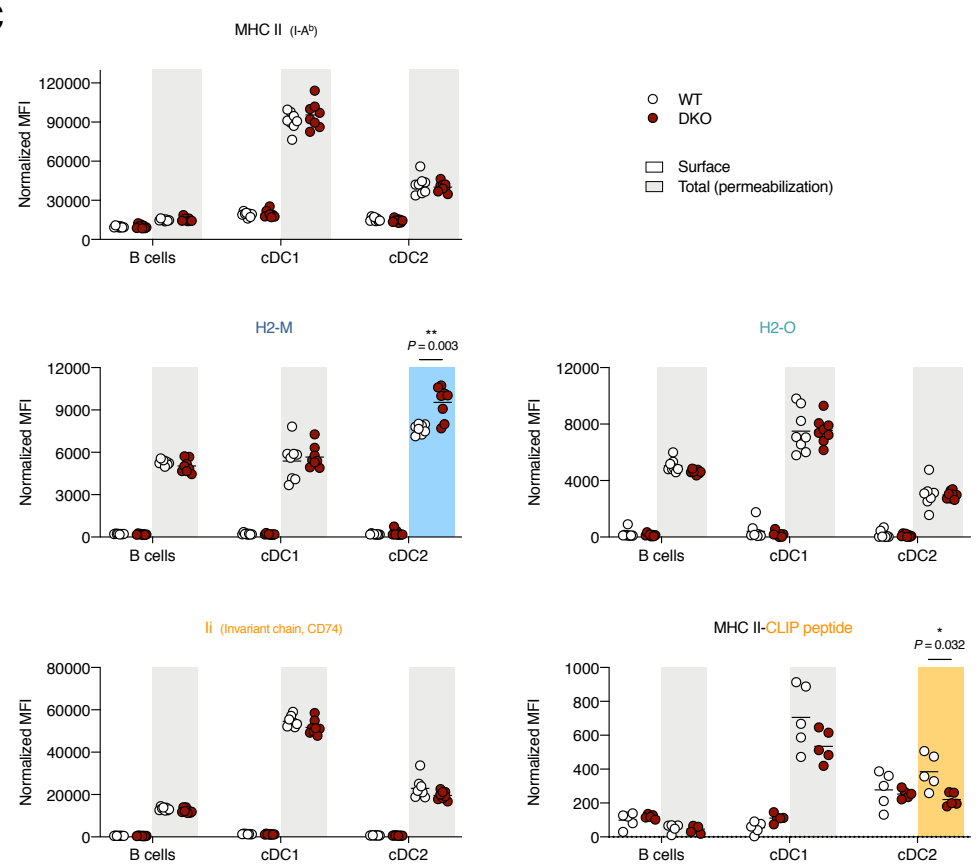
Figure 7

A

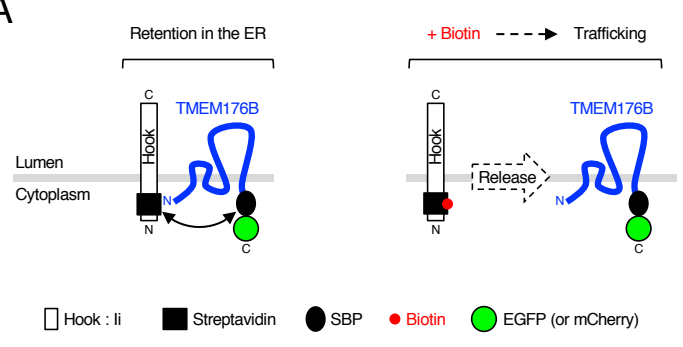

B

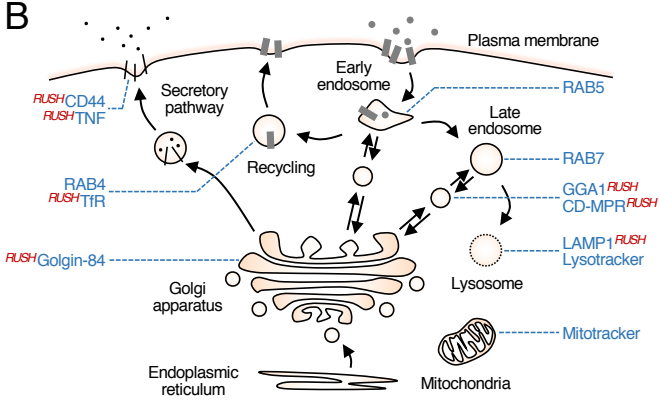

C

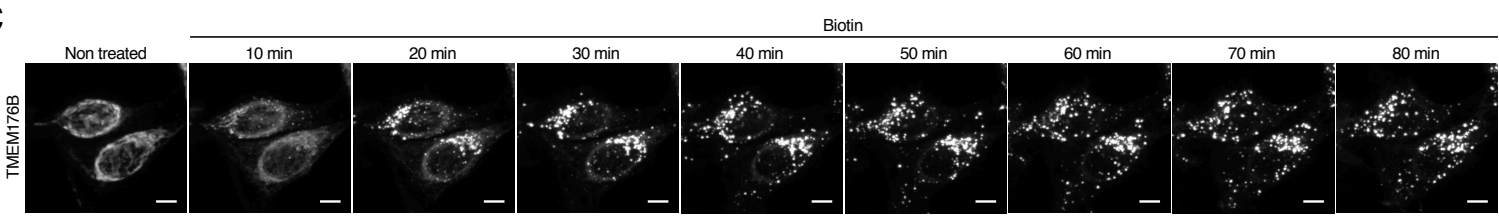

D

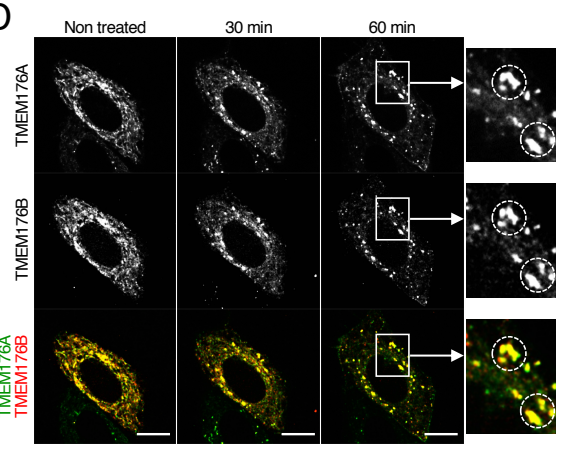

E

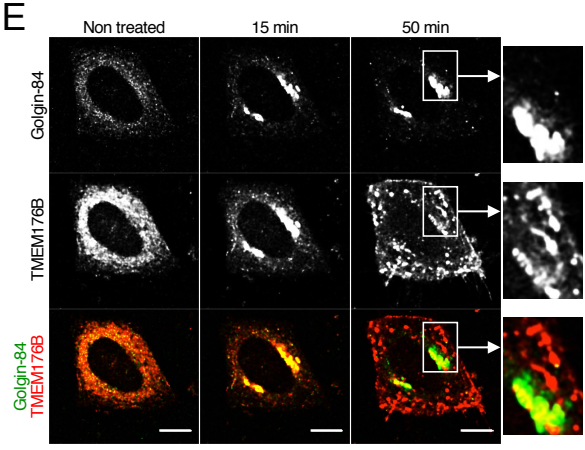

F

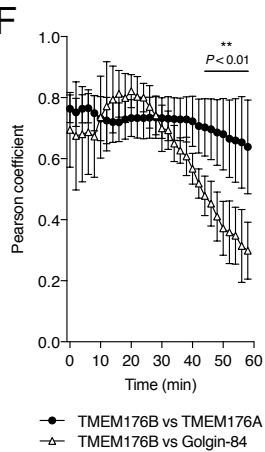

G
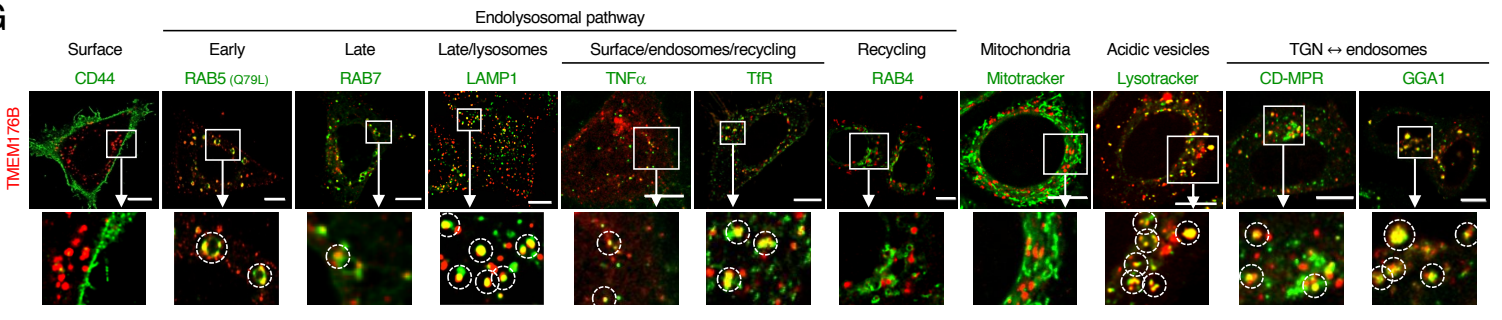

$\mathrm{H}$
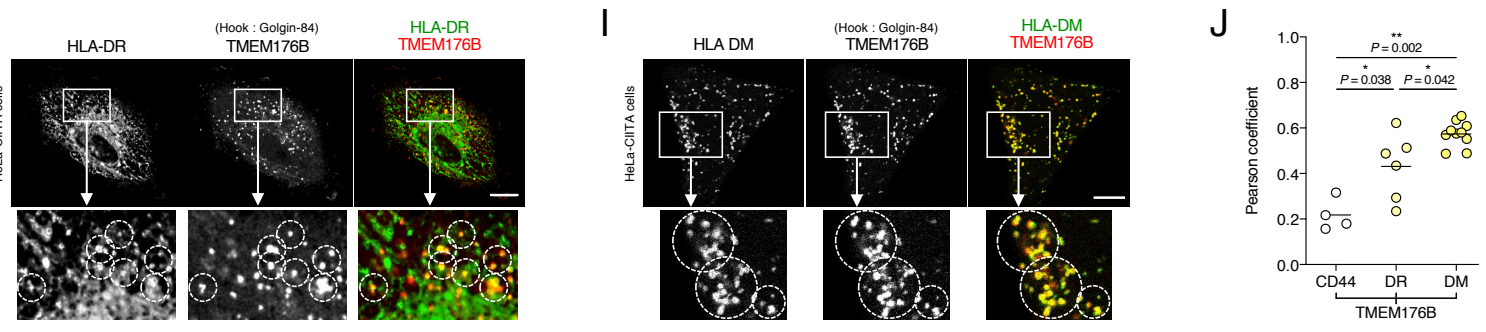